\title{
Charged-current muonic reactions in core-collapse supernovae
}

\author{
Gang Guo®, ${ }^{1,2, *}$ Gabriel Martínez-Pinedo®, ${ }^{1,3}$ A. Lohs, ${ }^{1}$ and Tobias Fischer ${ }^{4}$ \\ ${ }^{1}$ GSI Helmholtzzentrum für Schwerionenforschung, Planckstraße 1, 64291 Darmstadt, Germany \\ ${ }^{2}$ Institute of Physics, Academia Sinica, Taipei, 11529, Taiwan \\ ${ }^{3}$ Institut für Kernphysik (Theoriezentrum), Technische Universität Darmstadt, \\ Schlossgartenstraße 2, 64298 Darmstadt, Germany \\ ${ }^{4}$ Institute for Theoretical Physics, University of Wrocław, 50-204 Wrocław, Poland
}

(Received 22 June 2020; accepted 16 July 2020; published 29 July 2020)

\begin{abstract}
The steady advance in core-collapse supernova simulations requires a more precise description of neutrino processes in hot and dense matter. In this work, we study the rates of charged-current (CC) weak processes with (anti)muons in supernova matter. At the relativistic mean field level, we derive results for the rates of $\mathrm{CC}$ neutrino-nucleon reactions, taking into account full kinematics, weak magnetism and pseudoscalar terms, and $q^{2}$-dependent nucleon form factors in the hadronic current. In addition to muonic semileptonic processes we also consider purely leptonic processes. In particular, we show that inverse muon decay can dominate the opacities for low energy $\nu_{\mu}$ and $\bar{\nu}_{e}$ at densities $\gtrsim 10^{13} \mathrm{~g} \mathrm{~cm}^{-3}$.
\end{abstract}

DOI: 10.1103/PhysRevD.102.023037

\section{INTRODUCTION}

The core-collapse of a massive star leads to the formation of a neutron star and the subsequent supernova explosion. Most of the gravitational binding energy of the neutron star is released in the form of neutrinos that in the neutrinodriven mechanism are responsible for the ejection of the stellar mantle [1]. Consequently, neutrino processes in hot and dense nuclear medium play crucial roles in many aspects of core-collapse supernovae (CCSNe), in particular for the explosion mechanism and the nucleosynthesis of heavy elements [2-5]. The successful explosion of corecollapse supernovae by the neutrino-driven mechanism in three-dimensional simulations has demonstrated the high relevance of neutrino physics as well as the necessity of an accurate description of neutrino transport in hot and dense protoneutron star (PNS) [6].

Neutrino processes in hot and dense matter have been well studied in the literature [7-22], and their impacts on CCSN simulations have also been extensively explored (see, e.g., Refs. [23-33]). However, neutrino CC processes considered for most of the studies are limited to the lightest charged lepton, i.e., $e^{ \pm}$. Due to a larger rest mass, the production of $\mu^{ \pm}$was thought to be highly suppressed and their role in SN dynamics was traditionally ignored.

\footnotetext{
*gangg23@gmail.com
}

Published by the American Physical Society under the terms of the Creative Commons Attribution 4.0 International license. Further distribution of this work must maintain attribution to the author(s) and the published article's title, journal citation, and DOI. Funded by SCOAP ${ }^{3}$.
Recently, the relevance of muons has been demonstrated in 2D SN simulations [34]. It showed that the formation of muons in SN matter softens the equation of state (EOS), leads to higher neutrino luminosities and mean energies, and therefore facilitates neutrino-driven explosions. It should be pointed out that the production of $\mu^{ \pm}$, especially the accumulation of net $\mu$-lepton number, is closely related to the CC reactions of $\nu_{\mu}\left(\bar{\nu}_{\mu}\right)$, which are created via thermal pair processes like $e^{-}+e^{-} \rightarrow \nu_{\mu}+\bar{\nu}_{\mu}$ and $N+N \rightarrow N+$ $N+\nu_{\mu}+\bar{\nu}_{\mu}$, and affected by neutrino transport in $\mathrm{SN}$ matter.

To be specific, the more abundant electrons, compared to positrons, can lead to an excess of $\mu^{-}$than $\mu^{+}$via leptonic weak processes like $\nu_{\mu} / \bar{\nu}_{e}+e^{-} \rightarrow \nu_{e} / \bar{\nu}_{\mu}+\mu^{-}$. This is also aided by the semileptonic process, $\nu_{\mu}+n \rightarrow p+\mu^{-}$, which is more favored than $\bar{\nu}_{\mu}+p \rightarrow n+\mu^{+}$since neutrons are in higher energy states than protons. Due to muon number conservation, the initial excess of $\mu^{-}$is compensated by an excess of $\bar{\nu}_{\mu}$. With a more abundant flux and a lower neutralcurrent (NC) scattering cross section with nucleon, more $\bar{\nu}_{\mu}$ diffuse out of the protoneutron star, leading to a gradual buildup of net muon number, i.e., muonization. The appearance of muonization not only affects the neutrino spectra of all flavors and enhances the explosion ability, but may also play a non-negligible role in the subsequent neutron star/ black hole formation [34].

In this work, we aim to study the reaction rates of all the relevant weak processes involving $\mu^{-}$or $\nu_{\mu}$ (see Table I), which are required as input in numerical simulations for a consistent description of muonization and neutrino transport. It has been found that weak magnetism can enhance/ suppress the neutrino/antineutrino opacities significantly 
and thus affects the neutrino spectra and SN dynamics [13]. A relativistic treatment of both full kinematics (nuclear recoil) and weak magnetism for $\mathrm{CC} \nu_{e}\left(\bar{\nu}_{e}\right)$ nucleon reactions have been studied [20,33], with its impact recently explored in symmetric CCSN simulation [33]. For semileptonic reactions involving $\mu^{ \pm}$with large energy-momenta transfer, pseudoscalar coupling term in the hadronic weak current, which is normally neglected for $\nu_{e}$ reactions, is found as important as weak magnetism. Besides, the effects of nucleon form factors become comparable as energy-momenta transfer increases and need to be considered on the same footing as weak magnetism and pseudoscalar corrections. Hence, we extend the formalisms presented in [33] to include weak magnetism, pseudoscalar term and $q^{2}$-dependent form factors in the hadronic current for CC $\nu_{\mu}$-nucleon reaction. Treating nucleons at the mean field level for the semileptonic processes, we obtain the opacities with full kinematics in a fully relativistic formalism. Correlations among nucleons beyond the mean field level have been investigated in Refs. [11,12,17,35-37] and are neglected in this work. Purely leptonic processes have already been studied in the literature [7,9]. We will compare them with the semileptonic processes and discuss their relevances to $\mu^{ \pm}$production and neutrino transport. As muonic processes also contribute to the opacity of $\bar{\nu}_{e}$ neutrinos we also update the description of inverse neutron decay that has recently been studied in Ref. [33]. We also note that the relevance of pions in hot dense nuclear matter has recently been explored and weak reactions involving pions and muons can be another important opacity source for $\nu_{\mu}$ and $\bar{\nu}_{\mu}$ [38]. Here we only focus on the standard muonic reactions listed in Table I and briefly mention the possible role of pionic reactions.

The paper is organized as follows. We present the formalisms to calculate the rates of purely leptonic processes in Sec. II and the rates of the semileptonic processes in Sec. III. In Sec. IV, we discuss the effects of weak magnetism, pseudoscalar term and form factors in semileptonic processes involving $\mu^{ \pm}$, and then compare the contributions from different leptonic and semileptonic processes. We come to the conclusion in Sec. VI.

TABLE I. Weak reactions with $\mu^{ \pm}$or $\nu_{\mu}$ considered in this work. We also consider inverse neutron decay as opacity source for $\bar{\nu}_{e}$.

\begin{tabular}{ll}
\hline \hline Leptonic reactions & Semileptonic reactions \\
\hline$\nu_{\mu}+e^{-} \rightarrow \nu_{\mu}+e^{-}(\mathrm{a})$ & $\nu_{\mu}+n \rightarrow \mu^{-}+p(\mathrm{f})$ \\
$\bar{\nu}_{\mu}+e^{-} \rightarrow \bar{\nu}_{\mu}+e^{-}(\mathrm{b})$ & $\bar{\nu}_{\mu}+p \rightarrow \mu^{+}+n(\mathrm{~g})$ \\
$\nu_{\mu}+e^{-} \rightarrow \nu_{e}+\mu^{-}(\mathrm{c})$ & $\bar{\nu}_{e}+p+e^{-} \rightarrow n(\mathrm{~h})$ \\
$\bar{\nu}_{e}+e^{-} \rightarrow \nu_{\mu}+\mu^{-}(\mathrm{d})$ & \\
$\nu_{\mu}+e^{-}+\bar{\nu}_{e} \rightarrow \mu^{-}(\mathrm{e})$ & \\
\hline \hline
\end{tabular}

\section{LEPTONIC REACTIONS}

\section{A. Scattering}

For neutrinos scattering with charged leptons, $\nu_{1}+l_{2}^{-} \rightarrow$ $\nu_{3}+l_{4}^{-}$, the spin-averaged matrix elements can be expressed in a general form as

$$
\begin{aligned}
\left\langle|\mathcal{M}|^{2}\right\rangle= & \lambda_{1}\left(p_{1} \cdot p_{2}\right)\left(p_{3} \cdot p_{4}\right)+\lambda_{2}\left(p_{1} \cdot p_{4}\right)\left(p_{2} \cdot p_{3}\right) \\
& +\lambda_{3}\left(p_{1} \cdot p_{3}\right),
\end{aligned}
$$

where $p_{1,2,3,4}$ are the four-momenta of $\nu_{1}$ (particle 1), $l_{2}$ (particle 2), $\nu_{3}$ (particle 3 ) and $l_{4}$ (particle 4), respectively. The coefficients $\lambda_{i}$ are shown in the Appendix $\mathrm{A}$ for different processes in Table I. The scattering kernel for this general reaction can be expressed as [8]

$$
\begin{aligned}
R^{\text {in }}\left(E_{1}, E_{3}, \mu\right)= & 2 \int \frac{d^{3} p_{2}}{(2 \pi)^{3}} \frac{d^{3} p_{4}}{(2 \pi)^{3}} \frac{\left\langle|\mathcal{M}|^{2}\right\rangle}{16 E_{1} E_{2} E_{3} E_{4}} \\
& \times(2 \pi)^{4} \delta^{(4)}\left(p_{1}+p_{2}-p_{3}-p_{4}\right) \\
& \times f_{2}\left(E_{2}\right)\left[1-f_{4}\left(E_{4}\right)\right] \\
= & \lambda_{1} R_{1}+\lambda_{2} R_{2}+\lambda_{3} R_{3},
\end{aligned}
$$

where $E_{i}$ are the relativistic energies, $f_{2,4}$ are the Fermi distribution functions of the charged leptons, $\mu=\cos \theta_{13}$ with $\theta_{13}$ the angle between $\boldsymbol{p}_{1,3}$, and $R_{1,2,3}$ are contributions from different terms of $\left\langle|\mathcal{M}|^{2}\right\rangle$ shown in Eq. (1). Due to rotational invariance, the scattering kernel only depends on the relative angle between the incoming and outgoing neutrinos. It can also be checked that both the matrix element and the phase space integral do not depend on the azimuthal angle.

The $R_{i}$ can be solved analytically up to a remaining integral over $E_{2}$ as [7,9,39-41]

$$
\begin{aligned}
R_{i}= & \frac{1}{16 \pi \Delta^{5}} \int_{E_{-}}^{\infty} d E_{2} f_{2}\left(E_{2}\right) \\
& \times\left[1-f_{4}\left(E_{4}\right)\right]\left(A_{i} E_{2}^{2}+B_{i} E_{2}+C_{i}\right) \\
= & \frac{1}{16 \pi \Delta^{5}}\left(A_{i} I_{2}+B_{i} I_{1}+C_{i} I_{0}\right),
\end{aligned}
$$

where $E_{-}$and $\Delta$ are given by

$$
\begin{aligned}
E_{-}= & \frac{1}{2}\left\{\left(E_{3}-E_{1}\right)(1+k)\right. \\
& \left.+\Delta \sqrt{\left[(1+k)^{2}+\frac{2 m_{2}^{2}}{E_{1} E_{3}(1-\mu)}\right]}\right\}, \\
\Delta= & \sqrt{E_{1}^{2}-2 E_{1} E_{3} \mu+E_{3}^{2}},
\end{aligned}
$$


with

$$
k=\frac{Q}{E_{1} E_{3}(1-\mu)}, \quad Q=\frac{1}{2}\left(m_{4}^{2}-m_{2}^{2}\right) .
$$

Similarly, the coefficients $A_{i}, B_{i}$, and $C_{i}$ are all functions of $E_{1,3}, \mu$ and $m_{2,4}$, and are presented in Appendix A. The functions $I_{s=0,1,2}$ are defined as

$$
I_{s}=\int_{E_{-}}^{\infty} d E_{2} E_{2}^{s} f_{2}\left(E_{2}\right)\left[1-f_{4}\left(E_{1}+E_{2}-E_{3}\right)\right],
$$

and they can be expressed in terms of the Fermi-Dirac integrals which are more convenient to compute numerically,

$$
\begin{aligned}
I_{0}= & T \hat{I}_{0}\left(\eta^{\prime}, \eta, y\right) \\
= & T f_{\gamma}\left(\eta^{\prime}-\eta\right)\left[F_{0}\left(\eta^{\prime}-y\right)-F_{0}(\eta-y)\right], \\
I_{1}= & T^{2} \hat{I}_{1}\left(\eta^{\prime}, \eta, y\right) \\
= & T^{2} f_{\gamma}\left(\eta^{\prime}-\eta\right)\left\{\left[F_{1}\left(\eta^{\prime}-y\right)-F_{1}(\eta-y)\right]\right. \\
& \left.+y\left[F_{0}\left(\eta^{\prime}-y\right)-F_{0}(\eta-y)\right]\right\}, \\
I_{2}= & T^{3} \hat{I}_{2}\left(\eta^{\prime}, \eta, y\right) \\
= & T^{3} f_{\gamma}\left(\eta^{\prime}-\eta\right)\left\{\left[F_{2}\left(\eta^{\prime}-y\right)-F_{2}(\eta-y)\right]\right. \\
& +2 y\left[F_{1}\left(\eta^{\prime}-y\right)-F_{1}(\eta-y)\right] \\
& \left.+y^{2}\left[F_{0}\left(\eta^{\prime}-y\right)-F_{0}(\eta-y)\right]\right\},
\end{aligned}
$$

where the Fermi-Dirac integrals $F_{n}(z)$ and the function $f_{\gamma}(z)$ are defined by

$$
\begin{gathered}
F_{n}(z)=\int_{0}^{\infty} d x \frac{x^{n}}{\exp (x-z)+1}, \\
f_{\gamma}(z)=\frac{1}{\exp (z)-1},
\end{gathered}
$$

and the coefficients $y, \eta$, and $\eta^{\prime}$ are given by

$$
y=\frac{E_{-}}{T}, \quad \eta=\frac{\mu_{2}}{T}, \quad \eta^{\prime}=\frac{E_{3}-E_{1}+\mu_{4}}{T},
$$

with $T$ the temperature and $\mu_{2,4}$ the relativistic chemical potentials including the rest mass. Note that $F_{0}(z)$ has an exact expression $\ln \left(1+e^{z}\right)$. For $n \geq 1$, there are approximate expressions available for $F_{n}(z)$ which are valid for $z \gg 0$ and $z \ll 0$ and can reproduce the exact results within $20 \%$ at $z \approx 0[42,43]$. In this work we calculate $F_{n}(n \geq 1)$ numerically. We also note that $f_{\gamma}\left(\eta^{\prime}-\eta\right)$ in Eq. (9) is divergent for elastic scattering with $\eta=\eta^{\prime}$. For this special case, we have

$$
I_{0}=\left.T F_{0}^{\prime}(z)\right|_{z=\eta-y}
$$

$$
\begin{gathered}
I_{1}=\left.T^{2}\left[F_{1}^{\prime}(z)+y F_{0}^{\prime}(z)\right]\right|_{z=\eta-y}, \\
I_{2}=T^{3}\left[F_{2}^{\prime}(z)+2 y F_{1}^{\prime}(z)+y^{2} F_{0}^{\prime}(z)\right]_{z=\eta-y},
\end{gathered}
$$

where $F_{n}^{\prime}(z) \equiv d F_{n}(z) / d z$. For $n \geq 1, F_{n}^{\prime}(z)=n F_{n-1}(z)$.

Once the scattering kernels are known, neutrino opacity or inverse mean free path due to scattering with leptons can be obtained by integrating over the phase space of the finalstate neutrino as

$$
\chi\left(E_{1}\right)=\int \frac{d^{3} p_{3}}{(2 \pi)^{3}} R\left(E_{1}, E_{3}, \mu\right)\left[1-f_{3}\left(E_{3}\right)\right] .
$$

The scattering kernel for the inverse process, $\nu_{1}+l_{2}^{-} \leftarrow$ $\nu_{3}+l_{4}^{-}$, can be obtained via detailed balance:

$$
R^{\text {out }}\left(E_{1}, E_{3}, \mu\right)=\exp \left\{\frac{E_{3}-E_{1}-\Delta \mu_{24}}{T}\right\} R^{\text {in }}\left(E_{1}, E_{3}, \mu\right)
$$

with $\Delta \mu_{24}=\mu_{2}-\mu_{4}$.

\section{B. Inverse muon decay}

For inverse decay, we consider a process like $\nu_{1}+l_{2}+$ $\nu_{3} \rightarrow l_{4}$ in which two neutrinos are absorbed. Similarly to scattering, the matrix elements can be expressed as

$$
\begin{aligned}
\left\langle|\mathcal{M}|^{2}\right\rangle= & \lambda_{A 1}\left(p_{1} \cdot p_{2}\right)\left(p_{3} \cdot p_{4}\right)+\lambda_{A 2}\left(p_{1} \cdot p_{4}\right)\left(p_{2} \cdot p_{3}\right) \\
& +\lambda_{A 3}\left(p_{1} \cdot p_{3}\right)
\end{aligned}
$$

where the coefficients $\lambda_{A 1, A 2, A 3}$ are given in Appendix A.

The kernel for inverse decay can be written as $R_{A}\left(E_{1}, E_{3}, \mu\right)=\lambda_{A 1} R_{A 1}+\lambda_{A 2} R_{A 2}+\lambda_{A 3} R_{A 3}$, and $R_{A i}$ are given by

$$
\begin{aligned}
& R_{A i}= \frac{1}{16 \pi \Delta_{A}^{5}} \int_{E_{A-}}^{E_{A+}} d E_{2} f_{2}\left(E_{2}\right)\left[1-f_{4}\left(E_{4}\right)\right] \\
& \times\left(A_{A i} E_{2}^{2}+B_{A i} E_{2}+C_{A i}\right) \Theta\left(k-k_{0}\right) \\
&=\frac{1}{16 \pi \Delta_{A}^{5}}\left(A_{A i} I_{A 2}+B_{A i} I_{A 1}+C_{A i} I_{A 0}\right) \Theta\left(k-k_{0}\right),
\end{aligned}
$$

where

$$
\begin{aligned}
E_{A \pm}= & \frac{1}{2}\left\{\left(E_{3}+E_{1}\right)(k-1)\right. \\
& \left. \pm \Delta_{A} \sqrt{\left[(1-k)^{2}-\frac{2 m_{2}^{2}}{E_{1} E_{3}(1-\mu)}\right]}\right\}, \\
\Delta_{A}= & \sqrt{E_{1}^{2}+2 E_{1} E_{3} \mu+E_{3}^{2}} \\
k_{0}= & \frac{m_{2}+m_{4}}{m_{4}-m_{2}}
\end{aligned}
$$


with $k$ and $Q$ given in Eq. (5). The coefficients $A_{A i}, B_{A i}$, and $C_{A i}$ are presented in Appendix A, and

$$
\begin{aligned}
I_{A 0} & =T\left[\hat{I}_{0}\left(\eta_{A}^{\prime}, \eta, y_{-}\right)-\hat{I}_{0}\left(\eta_{A}^{\prime}, \eta, y_{+}\right)\right], \\
I_{A 1} & =T^{2}\left[\hat{I}_{1}\left(\eta_{A}^{\prime}, \eta, y_{-}\right)-\hat{I}_{1}\left(\eta_{A}^{\prime}, \eta, y_{+}\right)\right], \\
I_{A 2} & =T^{3}\left[\hat{I}_{2}\left(\eta_{A}^{\prime}, \eta, y_{-}\right)-\hat{I}_{2}\left(\eta_{A}^{\prime}, \eta, y_{+}\right)\right],
\end{aligned}
$$

with $\hat{I}_{0,1,2}$ introduced in Eq. (7) and $y_{ \pm}, \eta$, and $\eta_{A}^{\prime}$ given by $y_{ \pm}=\frac{E_{A \pm}}{T}, \quad \eta=\frac{\mu_{2}}{T}, \quad \eta_{A}^{\prime}=\frac{-E_{3}-E_{1}+\mu_{4}}{T}$.

Opacity for $\nu_{1}$ due to inverse decay can be expressed as

$$
\chi\left(E_{1}\right)=\int \frac{d^{3} p_{3}}{(2 \pi)^{3}} R_{A}\left(\mu, E_{1}, E_{3}\right) f_{3}\left(E_{3}\right) .
$$

Similarly, one can obtain the opacity for $\nu_{3}$ by replacing $d^{3} p_{3}$ with $d^{3} p_{1}$ and $f_{1}\left(E_{1}\right)$ with $f_{3}\left(E_{3}\right)$ in the above equation.

The kernel for the decay process, $l_{4} \rightarrow \nu_{1}+l_{2}+\nu_{3}$, can be obtained from detailed balance:

$$
R_{D}\left(E_{1}, E_{3}, \mu\right)=\exp \left\{-\frac{E_{1}+E_{3}+\Delta \mu_{24}}{T}\right\} R_{A}\left(E_{1}, E_{3}, \mu\right) .
$$

\section{SEMILEPTONIC REACTIONS}

\section{A. Neutrino absorption}

We consider semileptonic CC reactions of the form

$$
\nu_{1}+N_{2} \rightarrow l_{3}+N_{4},
$$

where $N_{2,4}$ stand for the initial and final state nucleons.

We take relativistic dispersion relations for nucleons in the nuclear medium, which can be parametrized in terms of effective masses $m^{*}$ and mean field potentials $U$ as

$$
E_{2,4}=\sqrt{m_{2,4}^{* 2}+\left|\boldsymbol{p}_{2,4}\right|^{2}}+U_{2,4}
$$

with the four-momentum of nucleons $p_{2,4}=\left(E_{2,4}, \boldsymbol{p}_{2,4}\right)$. The interaction potentials and effective masses can be deduced from relativistic mean field theory, based on which equation of state $(\mathrm{EoS})$ of hot and dense nuclear matter can be derived [44-54]. The nuclear EoS can also be studied based on nonrelativistic parameterizations of the nuclear potentials, such as the widely used Lattimer and Swesty (LS) EoS [55]. In this case, nucleons take nonrelativistic energy-momentum relation as $E_{\mathrm{NR}}=$ $|\boldsymbol{p}|^{2} /\left(2 m^{*}\right)+U$. When transferred to relativistic form,
$E \simeq \sqrt{m^{* 2}+|\boldsymbol{p}|^{2}}+m-m^{*}+U$. Note that within the LS EoS, the Landau effective masses of nucleons are simply the bare masses.

For the energies considered, the neutrino-nucleon interaction is described by a current-current interaction

$$
\mathcal{L}=\frac{G}{\sqrt{2}} l_{\mu} j^{\mu}
$$

where $G=G_{\mathrm{F}} V_{u d}$ for CC processes and $G=G_{\mathrm{F}}$ for NC processes, with $G_{\mathrm{F}}$ the Fermi coupling constant and $V_{u d}$ the up-down entry of the Cabibbo-Kobayashi-Maskawa matrix. The leptonic current is

$$
l_{\mu}=\bar{l}_{3} \gamma_{\mu}\left(1-\gamma_{5}\right) \nu_{1},
$$

and the effective hadronic current takes a general form,

$$
\begin{aligned}
j^{\mu}= & \bar{\psi}_{4}\left\{\gamma^{\mu}\left[G_{V}\left(q^{2}\right)-G_{A}\left(q^{2}\right) \gamma^{5}\right]\right. \\
& \left.+\frac{i F_{2}\left(q^{2}\right)}{2 M_{N}} \sigma^{\mu \nu} q_{\nu}^{*}-\frac{G_{P}\left(q^{2}\right)}{M_{N}} \gamma^{5} q^{* \mu}\right\} \psi_{2},
\end{aligned}
$$

where $\psi_{2,4}$ are the Dirac spinors of nucleons, $q=p_{1}-$ $p_{3}=p_{4}-p_{2}$ is the momentum transferred to the nucleon, and $M_{N}$ is the nucleon mass taken to be the average nucleon bare mass with $M_{N}=\left(m_{n}+m_{p}\right) / 2$. Note that for weak magnetism and pseudoscalar term, we introduce $q^{*}=p_{4}^{*}-p_{2}^{*}$ with $p_{2,4}^{*}=\left(E_{2,4}^{*}, \boldsymbol{p}_{2,4}\right)=$ $\left(\sqrt{\left|\boldsymbol{p}_{2,4}\right|^{2}+m_{2,4}^{* 2}}, \boldsymbol{p}_{2,4}\right)$ as required by conservation of the weak vector current $[20,33,56,57]$. The vector term, axial vector term, weak magnetism as well as pseudoscalar term, are all characterized by $q^{2}$-dependent coupling strengths or form factors as

$$
\begin{aligned}
G_{V}\left(q^{2}\right) & =\frac{g_{V}\left[1-\frac{q^{2}\left(\gamma_{p}-\gamma_{n}\right)}{4 M_{N}^{2}}\right]}{\left(1-\frac{q^{2}}{4 M_{N}^{2}}\right)\left(1-\frac{q^{2}}{M_{V}^{2}}\right)^{2}}, \\
G_{A}\left(q^{2}\right) & =\frac{g_{A}}{\left(1-\frac{q^{2}}{M_{A}^{2}}\right)^{2}}, \\
F_{2}\left(q^{2}\right) & =\frac{\gamma_{p}-\gamma_{n}-1}{\left(1-\frac{q^{2}}{4 M_{N}^{2}}\right)\left(1-\frac{q^{2}}{M_{V}^{2}}\right)^{2}}, \\
G_{P}\left(q^{2}\right) & =\frac{2 M_{N}^{2} G_{A}\left(q^{2}\right)}{m_{\pi}^{2}-q^{2}},
\end{aligned}
$$

where $\gamma_{p, n}$ are the magnetic moments of protons and neutrons with $\gamma_{p} \simeq 2.793$ and $\gamma_{n} \simeq-1.913$, the vector and axial vector coupling constants are $g_{V}=1$ and $g_{A} \simeq 1.27$, and $M_{V} \simeq 840 \mathrm{MeV}, M_{A} \simeq 1 \mathrm{GeV}$, and $m_{\pi} \simeq$ $139.57 \mathrm{MeV}$ are the vector mass, the axial mass, and the 
pion mass, respectively. As commonly taken in the literature, the above form factors can be approximated by $g_{V, A}$, $\gamma_{p}-\gamma_{n}-1$ and $g_{P}=2 m_{N}^{2} g_{A} /\left(m_{\pi}^{2}-q^{2}\right)$ if the momentum transfer dependence can be neglected. Note that the pole in $g_{P}$ at $q^{2}=m_{\pi}^{2}$ can not be encountered for muonic reactions since $q^{2}=\left(p_{1}-p_{3}\right)^{2}=m_{\mu}^{2}-2 p_{1} \cdot p_{3}<m_{\mu}^{2}<m_{\pi}^{2}$. The relevance of pseudoscalar term for $\nu_{\mu}$ reactions can be understood since $q^{2}$ can get close to $m_{\mu}^{2}$, resulting in a large value of $g_{P}\left(q^{2}\right)$. We will explore the effects of these interaction terms and form factors later in this work.

Different from scattering process for which one needs to compute the scattering kernel, for neutrino absorption we are interested in the opacity or inverse mean free path: ${ }^{1}$

$$
\begin{aligned}
\chi\left(E_{1}\right)= & 2 \int \frac{d^{3} \boldsymbol{p}_{2}}{(2 \pi)^{3}} \int \frac{d^{3} \boldsymbol{p}_{3}}{(2 \pi)^{3}} \int \frac{d^{3} \boldsymbol{p}_{4}}{(2 \pi)^{3}} \frac{\left\langle|\mathcal{M}|^{2}\right\rangle}{16 E_{1} E_{2}^{*} E_{3} E_{4}^{*}} \\
& \times(2 \pi)^{4} \delta^{(4)}\left(p_{1}+p_{2}-p_{3}-p_{4}\right) \\
& \times f_{2}\left(1-f_{3}\right)\left(1-f_{4}\right),
\end{aligned}
$$

where the square of the amplitude can be written as

$$
\begin{aligned}
\left\langle|\mathcal{M}|^{2}\right\rangle= & \left\langle|\mathcal{M}|^{2}\right\rangle_{V V} \pm\left\langle|\mathcal{M}|^{2}\right\rangle_{V A}+\left\langle|\mathcal{M}|^{2}\right\rangle_{A A} \\
& +\left\langle|\mathcal{M}|^{2}\right\rangle_{V F} \pm\left\langle|\mathcal{M}|^{2}\right\rangle_{A F}+\left\langle|\mathcal{M}|^{2}\right\rangle_{F F} \\
& +\left\langle|\mathcal{M}|^{2}\right\rangle_{A P}+\left\langle|\mathcal{M}|^{2}\right\rangle_{P P},
\end{aligned}
$$

corresponding to contributions from the vector $(V)$, axial vector $(A)$, weak magnetism $(F)$, pseudoscalar $(P)$ terms, and their interferences. They are functions of momenta of the initial and the final particles, see Appendix B. The "+”(“-") sign applies to neutrino(antineutrino) absorption, which is required by $C P T$ symmetry. As demonstrated in Ref. [13], the $A F$ interference term, $\pm\left\langle|\mathcal{M}|^{2}\right\rangle_{A F}$, increases (decreases) the neutrino (antineutrino) opacities. The $A P$ interference term, $\left\langle|\mathcal{M}|^{2}\right\rangle_{A P}$, contributes negatively, leading to a suppressed opacity for both neutrinos and antineutrinos. Note that there is no interference between $P$ and $V / F$ terms.

To obtain $\chi\left(E_{\nu}\right)$ for neutrino transport, we can either first integrate out all the angles analytically in Eq. (28), and then do a remaining 2D integral over energies numerically [41], or directly perform a 4D numerical integration after applying the energy-momentum $\delta$-function without any further analytical integration. We will do both ways independently, and show they give rise to similar results within $\sim 10^{-3}$ for all the conditions considered.

\section{1. $4 D$ integrals}

In the 4D integrals, we choose $E_{2},|\boldsymbol{q}|=\left|\boldsymbol{p}_{4}-\boldsymbol{p}_{2}\right|$, $\cos \theta_{q 2}$ and $\phi_{q 2}$ as the integration variables, where $\theta_{q 2}$ and $\phi_{q 2}$ are the polar and azimuthal angles of $\boldsymbol{p}_{2}$ with respect to $\boldsymbol{q}$. The opacity due to absorption on nucleons can be expressed as

$$
\begin{aligned}
\chi\left(E_{1}\right)= & 2 \int d \phi_{q 2} \int \cos \theta_{q 2} \int d|\boldsymbol{q}| \int d E_{2}\left\langle|\mathcal{M}|^{2}\right\rangle \\
& \times f_{2}\left(1-f_{3}\right)\left(1-f_{4}\right) \frac{|\boldsymbol{q}|\left|\boldsymbol{p}_{2}\right|}{16(2 \pi)^{4} E_{1}^{2} E_{4}^{*}} .
\end{aligned}
$$

The determination of the integration bounds is discussed in Appendix C 1. In this work, all the 4D integrals are solved via a Monte Carlo algorithm encoded in the CUBA library [58].

\section{2. $2 D$ integrals}

To perform the integration over angles analytically, one can sort the total matrix elements into different fourmomenta products:

$$
\begin{aligned}
\left\langle|\mathcal{M}|^{2}\right\rangle= & \mathcal{A} M_{\mathcal{A}}+\mathcal{B} M_{\mathcal{B}}+\cdots+\mathcal{K}_{\text {tot }} M_{\mathcal{K}}+\mathcal{L}_{\text {tot }} M_{\mathcal{L}}=(4 G)^{2}\left[\mathcal{A}\left(p_{1} \cdot p_{2}^{*}\right)\left(p_{3} \cdot p_{4}^{*}\right)\right. \\
& +\mathcal{B}\left(p_{1} \cdot p_{4}^{*}\right)\left(p_{3} \cdot p_{2}^{*}\right)+\mathcal{C}\left(p_{1} \cdot p_{2}^{*}\right)^{2}\left(p_{1} \cdot p_{3}\right)+\mathcal{D}\left(p_{1} \cdot p_{2}^{*}\right)\left(p_{1} \cdot p_{3}\right)^{2}+\mathcal{E}\left(p_{1} \cdot p_{2}^{*}\right)^{2} \\
& \left.+\mathcal{F}_{\text {tot }}\left(p_{1} \cdot p_{3}\right)^{2}+\mathcal{H}\left(p_{1} \cdot p_{2}^{*}\right)\left(p_{1} \cdot p_{3}\right)+\mathcal{J}_{\text {tot }}\left(p_{1} \cdot p_{2}^{*}\right)+\mathcal{K}_{\text {tot }}\left(p_{1} \cdot p_{3}\right)+\mathcal{L}_{\text {tot }}\right]
\end{aligned}
$$

with

$$
\begin{aligned}
& \mathcal{F}_{\text {tot }}=\mathcal{F}+\frac{\mathcal{F}^{P P}}{\left(m_{\pi}^{2}-q^{2}\right)^{2}}, \quad \mathcal{J}_{\text {tot }}=\mathcal{J}+\frac{\mathcal{J}^{A P}}{m_{\pi}^{2}-q^{2}}, \\
& \mathcal{K}_{\text {tot }}=\mathcal{K}+\frac{\mathcal{K}^{A P}}{m_{\pi}^{2}-q^{2}}+\frac{\mathcal{K}^{P P}}{\left(m_{\pi}^{2}-q^{2}\right)^{2}}, \quad \mathcal{L}_{\text {tot }}=\mathcal{L}+\frac{\mathcal{L}^{A P}}{m_{\pi}^{2}-q^{2}}+\frac{\mathcal{L}^{P P}}{\left(m_{\pi}^{2}-q^{2}\right)^{2}} .
\end{aligned}
$$

Neglecting firstly the form factor dependences, the coefficients $\mathcal{A}, \mathcal{B}, \ldots, \mathcal{K}_{\text {tot }}$, and $\mathcal{L}_{\text {tot }}$ are independent of momenta (see Appendix B), and can be taken out of the integral. With the above notation, the opacity can be expressed as

\footnotetext{
${ }^{1}$ We note that in Eq. (5) of our previous paper [33], $E_{2,4}$ should be replaced by $E_{2,4}^{*}$. However, they were correctly treated in our numerical calculations and studies therein are unaffected.
} 


$$
\begin{aligned}
\chi\left(E_{1}\right)= & \frac{G^{2}}{4 \pi^{3}} \frac{1}{E_{1}^{2}} \int_{E_{3-}}^{E_{3+}} d E_{3} \int_{E_{2-}}^{E_{2+}} d E_{2} f_{2}\left(1-f_{3}\right)\left(1-f_{4}\right) \\
& \times\left[\mathcal{A} I_{\mathcal{A}}+\mathcal{B} I_{\mathcal{B}}+\cdots+\mathcal{E} I_{\mathcal{E}}+\mathcal{H} I_{\mathcal{H}}\right. \\
& +\left(\mathcal{F} I_{\mathcal{F}}+\mathcal{F}^{P P} I_{\mathcal{F}}^{P P}\right)+\left(\mathcal{J} I_{\mathcal{J}}+\mathcal{J}^{A P} I_{J}^{A P}\right) \\
& +\left(\mathcal{K} I_{\mathcal{K}}+\mathcal{K}^{A P} I_{\mathcal{K}}^{A P}+\mathcal{K}^{P P} I_{\mathcal{K}}^{P P}\right) \\
& \left.+\left(\mathcal{L} I_{\mathcal{L}}+\mathcal{L}^{A P} I_{\mathcal{L}}^{A P}+\mathcal{L}^{P P} I_{\mathcal{L}}^{P P}\right)\right]
\end{aligned}
$$

where $I_{\mathcal{X}}(\mathcal{X}=\mathcal{A}, \mathcal{B}, \ldots)$ are integrals over the angles and are given by

$$
\begin{aligned}
I_{\mathcal{X}}= & \frac{\bar{p}_{1} \bar{p}_{2} \bar{p}_{3} \bar{p}_{4}}{4 \pi^{2}} \int d \Omega_{2} d \Omega_{3} d \Omega_{4} d E_{4} M_{\mathcal{X}} \\
& \times \delta^{(4)}\left(p_{1}+p_{2}-p_{3}-p_{4}\right) \\
= & \frac{\bar{p}_{1} \bar{p}_{2} \bar{p}_{3} \bar{p}_{4}}{4 \pi^{2}} \int d \Omega_{2} d \Omega_{3} d \Omega_{4} M_{\mathcal{X}} \\
& \times \delta^{(3)}\left(\boldsymbol{p}_{1}+\boldsymbol{p}_{2}-\boldsymbol{p}_{3}-\boldsymbol{p}_{4}\right),
\end{aligned}
$$

with $\bar{p}_{i}=\left|\boldsymbol{p}_{i}\right|$ and $M_{\mathcal{X}}$ introduced in Eq. (31). Similarly, $I_{\mathcal{X}}^{A P, P P}$ are given by

$$
\begin{aligned}
I_{\mathcal{X}}^{A P}= & \frac{\bar{p}_{1} \bar{p}_{2} \bar{p}_{3} \bar{p}_{4}}{4 \pi^{2}} \int d \Omega_{2} d \Omega_{3} d \Omega_{4} \frac{M_{\mathcal{X}}}{m_{\pi}^{2}-q^{2}} \\
& \times \delta^{3}\left(\boldsymbol{p}_{1}+\boldsymbol{p}_{2}-\boldsymbol{p}_{3}-\boldsymbol{p}_{4}\right), \\
I_{\mathcal{X}}^{P P}= & \frac{\bar{p}_{1} \bar{p}_{2} \bar{p}_{3} \bar{p}_{4}}{4 \pi^{2}} \int d \Omega_{2} d \Omega_{3} d \Omega_{4} \frac{M_{\mathcal{X}}}{\left(m_{\pi}^{2}-q^{2}\right)^{2}} \\
& \times \delta^{3}\left(\boldsymbol{p}_{1}+\boldsymbol{p}_{2}-\boldsymbol{p}_{3}-\boldsymbol{p}_{4}\right) .
\end{aligned}
$$

All the $I_{\mathcal{X}}, I_{\mathcal{X}}^{A P, P P}$ can be computed analytically [41], and their expressions are presented in Appendix B. The bounds $E_{2 \pm}$ and $E_{3 \pm}$ can be obtained numerically from energymomenta conservation, and only the kinematically allowed regions contribute to the opacities, see Appendix C.

As mentioned above, for neutrinos with energies $E_{1} \gtrsim$ $100 \mathrm{MeV}$ or reactions involving $\nu_{\mu}\left(\bar{\nu}_{\mu}\right)$, the suppression of the rates due to form factor will become significant. Considering the $q^{2}$-dependent coupling constants, see Eq. (27), the analytical integrations over angles become nontrivial. One possible way is to expand the form factors in powers of $q^{2} / M_{V, A, N}^{2}$, and do the analytical angular integration with the leading-order corrections. However, we choose not to show the lengthy expressions in this work. ${ }^{2}$ Instead, we treat the form factor effects exactly in our 4D integral approach.

\footnotetext{
${ }^{2}$ In the provided subroutine for the $2 \mathrm{D}$ integrals, an approximate treatment of the form factor has been added. However, since we only consider the leading-order term, the resulting opacities may not be reliable for $E_{\nu} \gtrsim 100 \mathrm{MeV}$.
}

\section{B. Inverse neutron decay}

At the conditions relevant to SN matter, neutron is more massive and has a higher mean field potential than proton. Therefore, the only allowed decay/inverse decay is $n \rightleftarrows \bar{\nu}_{e}+p+e^{-}$. Despite that, we still choose to consider a general process as, $\nu_{1}+N_{2}+l_{3} \rightarrow N_{4}$, where same notations are taken as in Sec. III A. Note that the role of inverse neutron decay on supernova neutrinos has recently been explored [33].

The matrix element for the inverse decay, $\nu_{1}+N_{2}+$ $l_{3} \rightarrow N_{4}$, can be simply obtained from that for the capture, $\nu_{1}+N_{2} \rightarrow l_{3}+N_{4}$, with $\nu_{1}, l_{3}$ and $N_{2,4}$ the same particle species as in the inverse decay,

$$
|\mathcal{M}|_{\text {inverse }}^{2}\left(p_{1}, p_{2}, p_{3}, p_{4}\right)=|\mathcal{M}|_{\text {capture }}^{2}\left(p_{1}, p_{2},-p_{3}, p_{4}\right) .
$$

Similarly to captures, we can perform both the 4D and the $2 \mathrm{D}$ integrations. For the $4 \mathrm{D}$ integrals, we take the same integration variables as for the capture, i.e., $E_{2},|\boldsymbol{q}|, \cos \theta_{q 2}$, $\phi_{q 2}$, and the opacity due to inverse decay is given by

$$
\begin{aligned}
\chi\left(E_{1}\right)= & 2 \int d \phi_{q 2} \int \cos \theta_{q 2} \int d|\boldsymbol{q}| \int d E_{2}\left\langle|\mathcal{M}|^{2}\right\rangle \\
& \times f_{2} f_{3}\left(1-f_{4}\right) \frac{|\boldsymbol{q}|\left|\boldsymbol{p}_{2}\right|}{16(2 \pi)^{4} E_{1}^{2} E_{4}^{*}},
\end{aligned}
$$

with the bounds for inverse decay determined in Sec. C 2 .

Integrating out all the angles, the opacity can be expressed as a $2 \mathrm{D}$ integral:

$$
\begin{aligned}
\chi\left(E_{1}\right)= & \frac{G^{2}}{4 \pi^{3}} \frac{1}{E_{1}^{2}} \int_{E_{3-}}^{E_{3+}} d E_{3} \int_{E_{2-}}^{E_{2+}} d E_{2} f_{2} f_{3}\left(1-f_{4}\right) \\
& \times\left[\tilde{\mathcal{A}} \tilde{I}_{\mathcal{A}}+\tilde{\mathcal{B}} \tilde{I}_{\mathcal{B}}+\cdots+\tilde{\mathcal{E}}_{\mathcal{E}}+\tilde{\mathcal{H}} \tilde{I}_{\mathcal{H}}\right. \\
& +\left(\tilde{\mathcal{F}} \tilde{I}_{\mathcal{F}}+\tilde{\mathcal{F}}^{P P} \tilde{I}_{\mathcal{F}}^{P P}\right)+\left(\tilde{\mathcal{J}} \tilde{I}_{\mathcal{J}}+\tilde{\mathcal{J}}^{A P} \tilde{I}_{\mathcal{J}}^{A P}\right) \\
& +\left(\tilde{\mathcal{K}} \tilde{I}_{\mathcal{K}}+\tilde{\mathcal{K}}^{A P} \tilde{I}_{\mathcal{K}}^{A P}+\tilde{\mathcal{K}}^{P P} \tilde{I}_{\mathcal{K}}^{P P}\right) \\
& \left.+\left(\tilde{\mathcal{L}} \tilde{I}_{\mathcal{L}}+\tilde{\mathcal{L}}^{A P} \tilde{I}_{\mathcal{L}}^{A P}+\tilde{\mathcal{L}}^{P P} \tilde{I}_{\mathcal{L}}^{P P}\right)\right],
\end{aligned}
$$

where $\tilde{\mathcal{X}}, \tilde{\mathcal{X}}^{A P, P P}, \tilde{I}_{\mathcal{X}}$, and $\tilde{I}_{\mathcal{X}}^{A P, P P}$ can be obtained from the expressions for captures [see Eq. (33)] with the replacement

$$
\begin{aligned}
\left\{\tilde{\mathcal{X}}, \tilde{\mathcal{X}}^{A P, P P}\right\} & =\left.\left\{\mathcal{X}, \mathcal{X}^{A P, P P}\right\}\right|_{E_{3} \rightarrow-E_{3}}, \\
\left\{\tilde{I}_{\mathcal{X}}, \tilde{I}_{\mathcal{X}}^{A P, P P}\right\} & =-\left.\left\{I_{\mathcal{X}}, I_{\mathcal{X}}^{A P, P P}\right\}\right|_{E_{3} \rightarrow-E_{3}} .
\end{aligned}
$$

The threshold of $E_{1}$ and the integration bounds, $E_{2, \pm}$ and $E_{3, \pm}$, can be derived following the discussions in Sec. C 2 .

\section{RESULTS AND DISCUSSION}

Based on the formulas presented above, we can study the neutrino opacities from different processes. To proceed, we firstly show the consistency between the opacities of 
TABLE II. Two conditions considered in our study. The mean field potentials of nucleons are derived within the LattimerSwesty EoS, and nucleons take bare masses. All quantities are in units of $\mathrm{MeV}$.

\begin{tabular}{cccccccccr}
\hline \hline & \multirow{2}{*}{$\mu_{n}$} & $\mu_{p}$ & $U_{n}$ & $U_{p}$ & $\mu_{e}$ & $\mu_{\mu}$ & $\mu_{\nu_{e}}$ & \multicolumn{1}{c}{$\mu_{\nu_{\mu}}$} \\
\hline $\mathrm{A}$ & 38.3 & 886.0 & 800.7 & -24.9 & -42.9 & 83.3 & 64.1 & -2.1 & -20.0 \\
$\mathrm{~B}$ & 15.2 & 912.7 & 875.4 & -6.1 & -8.9 & 44.4 & 37.3 & 7.1 & 1.3 \\
\hline \hline
\end{tabular}

semileptonic processes from our 2D and 4D integrals, and then discuss the effects of weak magnetism, pseudoscalar coupling term as well the $q^{2}$-dependent form factors. We also compare the different leptonic and semileptonic processes at conditions relevant to CCSN matter.

For our studies, we take the profiles from a 2D simulation $[34,59]$ for a nonrotating $20 M_{\odot}$ progenitor star [60] based on the Lattimer-Swesty EOS (LS200) [55], where the relevant muonic reactions have been implemented. ${ }^{3}$ The chemical potentials of all particles and the interaction potential of nucleons required to obtain neutrino opacities are obtained consistently with the same EOS.

To better demonstrate the role of weak muonic processes on muonization, we take two conditions at $r \simeq 13.6 \mathrm{~km}$ (condition A) and at $20 \mathrm{~km}$ (condition B) at $0.4 \mathrm{~s}$ after core bounce, with $\rho \simeq 10^{14} \mathrm{~g} \mathrm{~cm}^{-3}$ and $T \simeq 38.3 \mathrm{MeV}$, and $\rho \simeq$ $1.3 \times 10^{13} \mathrm{~g} \mathrm{~cm}^{-3}$ and $T \simeq 15.2 \mathrm{MeV}$, respectively. The chemical potentials of all relevant particles as well as the mean field interaction potentials of nucleons derived based on LS200 are listed in Table II. The corresponding lepton fractions are $Y_{e}=0.13$ and $Y_{\mu}=0.04$ for condition A, and $Y_{e}=0.11$ and $Y_{\mu}=0.002$ for condition B.

\section{A. CC semileptonic processes}

The semileptonic processes with $\mu^{-}$production are only relevant when temperature is high enough or density is high enough so that the chemical potential difference of nucleons, $\mu_{n}-\mu_{p}$, or the interaction potential difference, $\Delta U_{n p}=U_{n}-U_{p}$, is comparable to the muon rest mass.

\section{Consistency between the 2D/4D integrals}

Figure 1 demonstrates the consistencies between our 4D and 2D integrals for $\nu_{\mu}+n \rightarrow \mu^{-}+p$ at condition $\mathrm{A}$, where contributions from $g_{A}^{2}, g_{V}^{2}$, and $g_{A} g_{V}$ terms (leadingorder term, black line), from $f_{2}^{2}, g_{V} f_{2}$, and $g_{A} f_{2}$ terms (weak magnetism, red line), and from $g_{P}^{2}$ and $g_{A} g_{P}$ terms (pseudoscalar term, green line) are considered separately. The nucleon form factors have not been considered. As shown in the figure, the two different methods lead to consistent opacities within $\sim 0.1 \%$. The fluctuations are arisen from the Monte Carlo sampling in the 4D integrals.

\footnotetext{
${ }^{3}$ Note that the CC semileptonic processes of $\nu_{\mu}$ in [34] were only approximately treated, not fully consistent with the formalisms presented in our work.
}

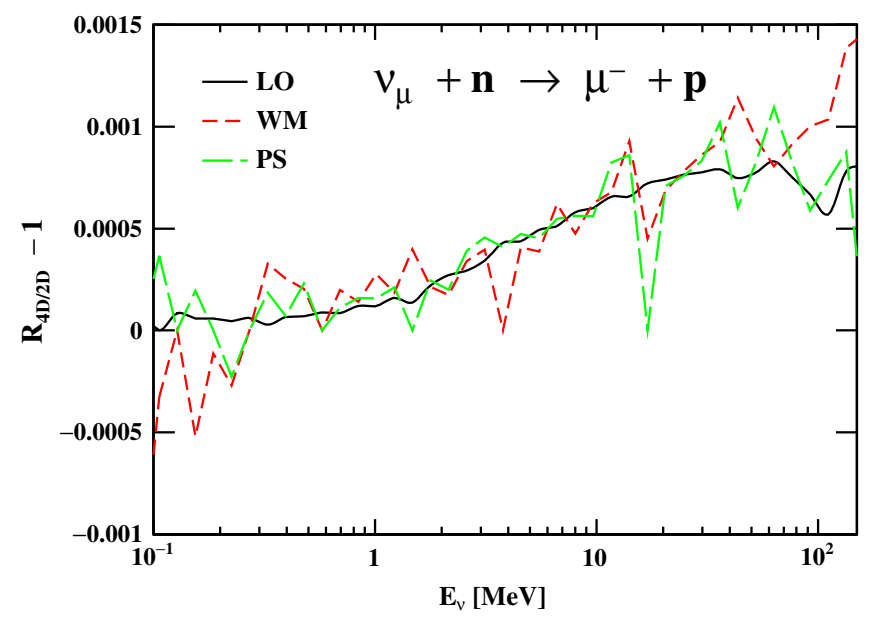

FIG. 1. $R_{4 \mathrm{D} / 2 \mathrm{D}}-1$ as functions of neutrino energy, $E_{\nu}$, with $R_{4 \mathrm{D} / 2 \mathrm{D}}$ the ratio of opacity from the $4 \mathrm{D}$ integral to that from the 2D integral for $\nu_{\mu}+n \rightarrow \mu^{-}+p$ at condition A. Contributions from leading-order terms (LO), weak magnetism (WM), and pseudoscalar terms (PS) are considered separately. Nucleon form factors have not been considered.

Although not shown here, we have checked such consistency also applies to the general conditions encountered in $\mathrm{SN}$ simulations. Typically, it is more efficient to use the 2D integrals than the 4D integrals. However, the 4D integral can provide a more straightforward calculation of the opacities without any tedious analytical derivations, and a robust check of the $2 \mathrm{D}$ results. Besides, the form factor effect can be included exactly in our 4D integrals, while the 2D integrals only treat it approximately. Without otherwise stated, we use results from the $4 \mathrm{D}$ integrals for the discussions below.

\section{Effects of weak magnetism, pseudoscalar, and form factors}

Figure 2 compares the effects of weak magnetism (WM), pseudoscalar term (PS), and form factors (FF) on the neutrino opacities for $\nu_{\mu}+n \rightarrow \mu^{-}+p$ at conditions A and B. As shown in the figure, weak magnetism enhances the absorption rates of $\nu_{\mu}$ by $\sim 20 \%$ at $E_{\nu}=20 \mathrm{MeV}$ and by $40 \%-50 \%$ at $E_{\nu}=150 \mathrm{MeV}$, which is consistent qualitatively with the studies in [13]. However, the correction factor due to weak magnetism introduced in [13] did not consider the finite charged lepton mass and the mass/ potential shifts of nucleons, and in general can not reproduce well our weak magnetism. One can in principle extend the analytical studies of [13], which assumes an initial neutron at rest and ignores the final state blocking, to incorporate the mean field effects as well as a finite muon mass. However, those approximations are not justified at the high density and temperature conditions considered in this work.

Including the pseudoscalar term suppresses the opacities for neutrino absorption on neutron since the interference 

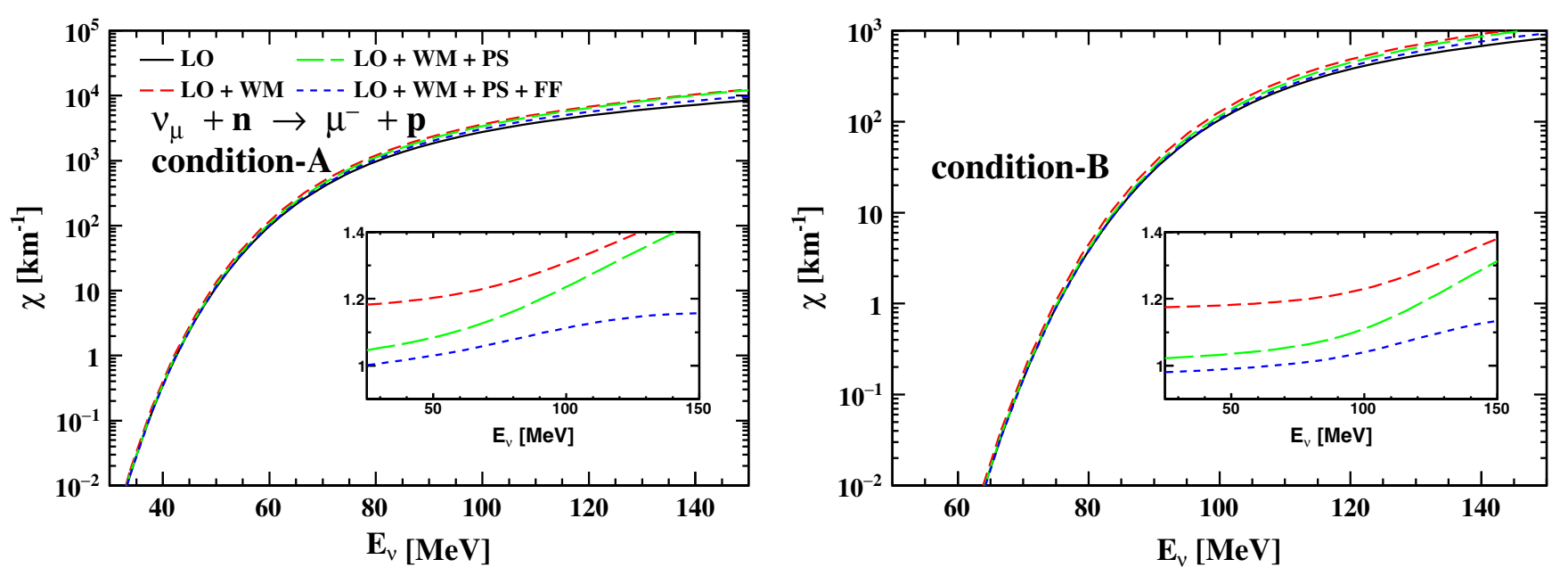

FIG. 2. Neutrino opacities for $\nu_{\mu}+n \rightarrow \mu^{-}+p$ and the effects of weak magnetism (WM), pseudoscalar term (PS), and form factors (FF) at conditions A and B. The relative ratios of the opacities to that considering only the leading-order terms (LO, the black lines), i.e., terms proportional to $g_{V}^{2}, g_{A}^{2}$ and $g_{V} g_{A}$, are shown in the inset as functions of $E_{\nu}$. Note that full kinematics including nucleon recoils are always considered.

term $\left\langle|\mathcal{M}|^{2}\right\rangle_{A P}$ is negative, which dominates over the positive term $\left\langle|\mathcal{M}|^{2}\right\rangle_{P P}$. The pseudoscalar term has a large impact at small $E_{\nu}$, which then decreases with increasing $E_{\nu}$. Such behavior can be understood as follows. Neglecting the mean field effects on nucleon masses and $\Delta U_{n p}^{2}$ terms, the matrix element $\left\langle|\mathcal{M}|^{2}\right\rangle_{A P}$ shown in Eq. (B1g) is proportional to $\left[-\left(p_{1} \cdot p_{3}\right)+2 E_{\nu} \Delta U_{n p}\right] m_{\mu}^{2}$ with $\left(p_{1} \cdot p_{3}\right) \sim m_{\mu}^{2}$. As $E_{\nu}$ increases, the second term becomes more relevant and cancels the first term. We also note that the pseudoscalar term has negligible effects on $\nu_{e}$ reactions as it scales with $m_{l}^{2}$. Differently from weak magnetism, the pseudoscalar term also suppresses the opacity for antineutrino absorption.

The reduction of opacities due to the $q^{2}$-dependent form factors is easy to understand since $q^{2}$ is always negative [see Eq. (27)]. As $\left|q^{2}\right|$ increases with $E_{\nu}$, the effects will become more significant. For $\nu_{\mu}$ absorption, the

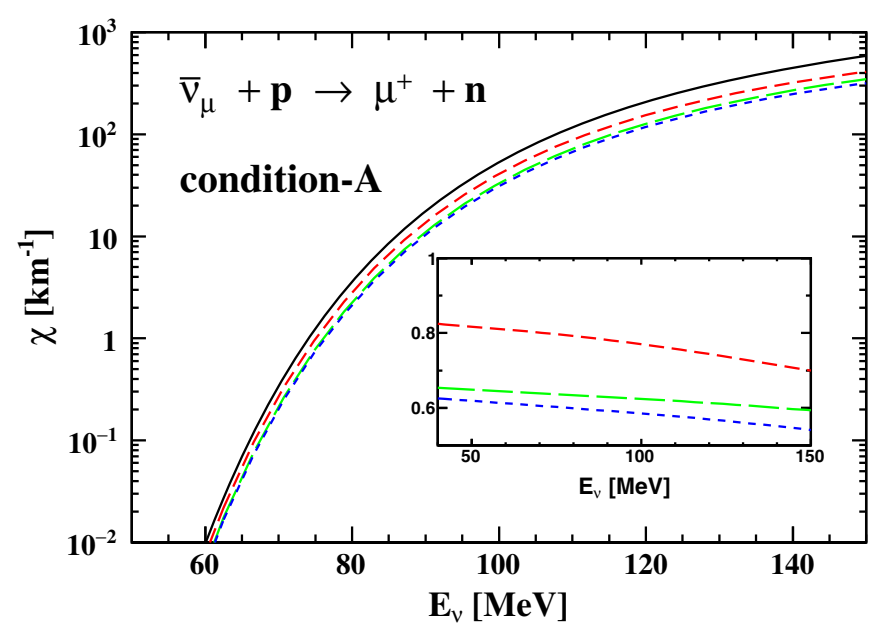

enhancement due to weak magnetism, of (20-30)\% at $E_{\nu}=100 \mathrm{MeV}$, is largely cancelled by the effects of pseudoscalar term and form factor. The net effect, compared to the leading-order results, is to enhance the opacities by only about $10 \%$ and $4 \%$ at $E_{\nu}=100 \mathrm{MeV}$ for conditions A and B, respectively. We have also checked that such cancellation is quite robust for conditions relevant to supernova matter. For the regions of interest, all effects combined enhance the opacities of $\nu_{\mu}$ of energies around $100 \mathrm{MeV}$ by $\lesssim 10 \%$ compared to the leading-order results.

As shown in Fig. 3, the opacities of $\bar{\nu}_{\mu}$ from $\bar{\nu}_{\mu}+p \rightarrow$ $\mu^{+}+n$ are suppressed by $\sim 40 \%$ for all the relevant $E_{\nu}$ at both conditions, as all effects contribute negatively. Since protons are in lower energy states compared to neutrons, the rates of $\bar{\nu}_{\mu}$ absorption on protons are about 30 times slower than those of $\nu_{\mu}$.

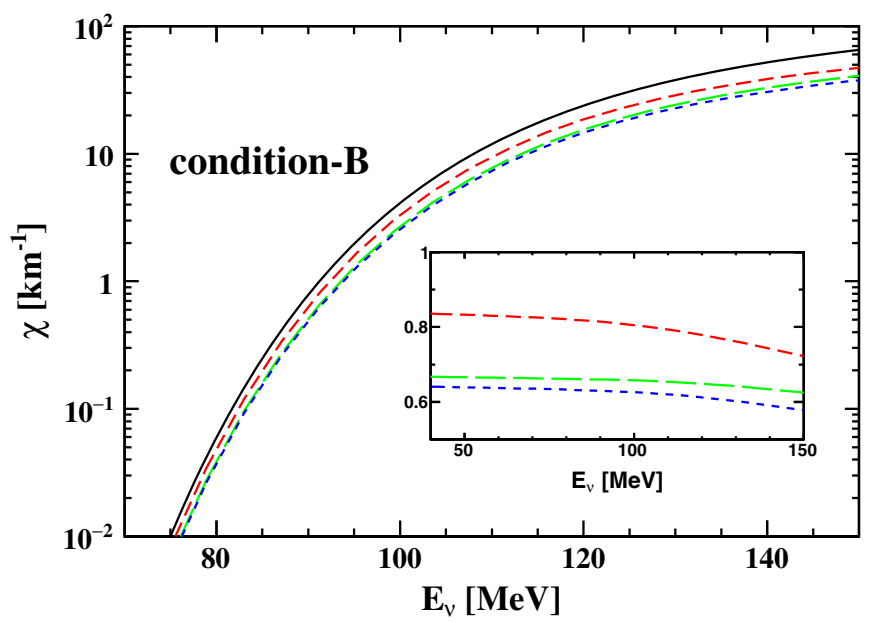

FIG. 3. Same as Fig. 2 but for $\bar{\nu}_{\mu}+p \rightarrow \mu^{+}+n$. 


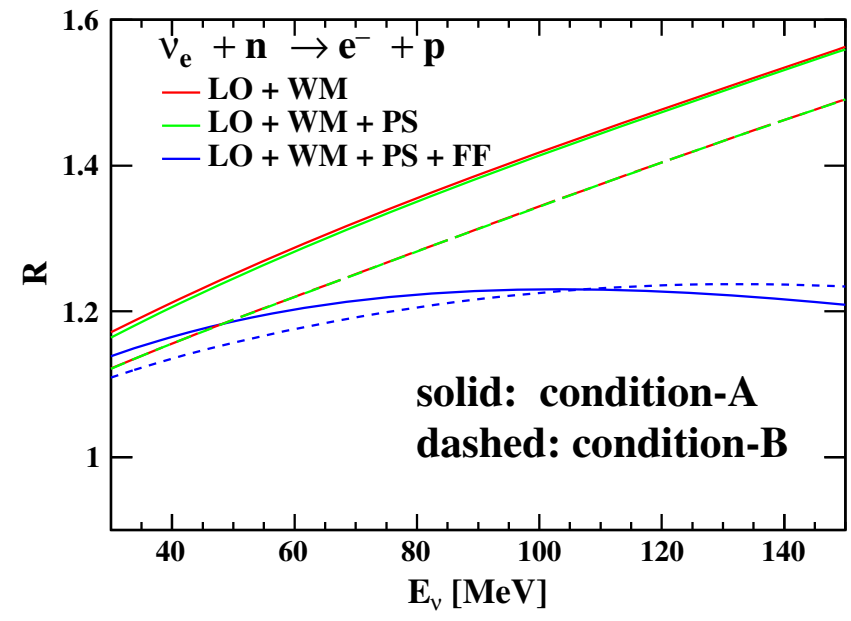

FIG. 4. Ratios of $\nu_{e}$ opacities for $\nu_{e}+n \rightarrow e^{-}+p$ including weak magnetism, pseudoscalar term, and form factor effects to the leading-order results.

The transport of $\nu_{e}$ is typically dominated by the CC absorption on neutrons in hot PNS. Therefore, an accurate description of this process is important. We show in Fig. 4 the effects of different treatments of the hadronic current for $\nu_{e}$. As mentioned above, the pseudoscalar term has negligible effects. However, the effect of form factors is nonnegligible at high $E_{\nu}$. It can suppress the $\nu_{e}$ absorption rates by (10-20)\% for $E_{\nu} \gtrsim 100 \mathrm{MeV}$. The net effect incorporating all corrections is to enhance the leading-order results by $\sim 20 \%$ at conditions $\mathrm{A}$ and $\mathrm{B}$.

\section{B. Muonization from leptonic and semileptonic processes}

Figure 5 compares the opacities of different weak muonic processes at conditions $\mathrm{A}$ and $\mathrm{B}$. There are mainly four weak processes contributing to $\mu^{-}$production: $\nu_{\mu}+$ $n \rightarrow \mu^{-}+p, \nu_{\mu}+e^{-} \rightarrow \mu^{-}+\nu_{e}, \bar{\nu}_{e}+e^{-} \rightarrow \bar{\nu}_{\mu}+\mu^{-}$, and $\nu_{\mu}+e^{-}+\bar{\nu}_{e} \rightarrow \mu^{-}$. The semileptonic processes always dominate at high $E_{\nu}$ due to larger matrix elements. At low $E_{\nu}$, purely leptonic reactions between $\nu_{\mu}$ and electrons become more important for $\mu^{-}$production due to a larger physically allowed phase space. Especially, inverse muon decay (blue short-dashed lines) allows neutrinos with energies below 10-20 MeV to contribute. With a FermiDirac distribution, the averaged energies of neutrinos are about $3.15 \times T$. For condition A with higher temperature and density $\left(\left\langle E_{\nu}\right\rangle \sim 100 \mathrm{MeV}\right)$, the semileptonic processes are the main production channel for $\mu^{ \pm}$, while at outer region with lower temperature (condition B with $\left\langle E_{\nu}\right\rangle \sim$ $50 \mathrm{MeV}$ ), leptonic processes are more relevant. Compared to $\mu^{-}$, the rates of $\mu^{+}$production via semileptonic or leptonic processes are slower by a factor of $\sim 30$, leading to a gradual buildup of $\mu^{-}$excess over $\mu^{+}$. Note that although the electromagnetic processes like $e^{-}+e^{+} \rightarrow$ $\mu^{-}+\mu^{+}$and $\gamma+\gamma \rightarrow \mu^{-}+\mu^{+}$are much faster than the weak processes, they do not contribute directly to muonization since $\mu^{ \pm}$are always produced in pairs. Considering a high electron chemical potential (with $\mu_{e} \approx 83 \mathrm{MeV}$ and $\approx 44 \mathrm{MeV}$ for conditions $\mathrm{A}$ and $\mathrm{B}$, respectively), $\mu^{-}$decay rate is lower than that of $\mu^{+}$due to final state $e^{-}$blocking, which could also play a role in muonization.

The electromagnetic processes should act fast enough to keep muons in local thermal equilibrium. Due to muon number conservation, muonization is further determined by $\nu_{\mu}$ and $\bar{\nu}_{\mu}$ transport, and specifically, their difference. As shown in Fig. 5, neutrino opacities are dominated by semileptonic processes with nucleons. For NC neutrinonucleon scattering (red dashed lines), we take the elastic approximation as in Ref. [8] and only consider contributions from scattering with neutrons. Inclusion of weak magnetism leads to a higher $\nu_{\mu}$ opacity from NC scattering with nucleons compared to that of $\bar{\nu}_{\mu}$ [13], and thus affects muonization [61]. The CC semileptonic reaction rates of $\nu_{\mu}$

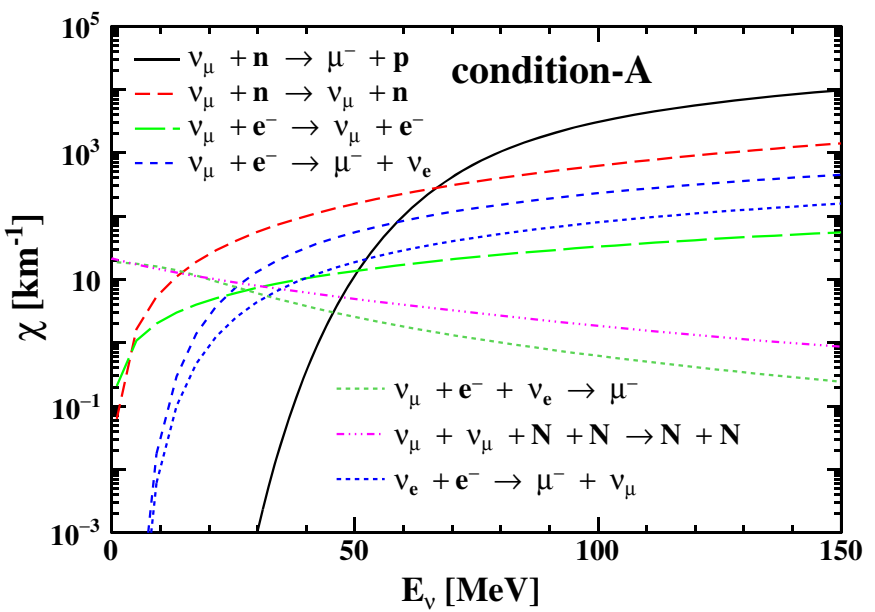

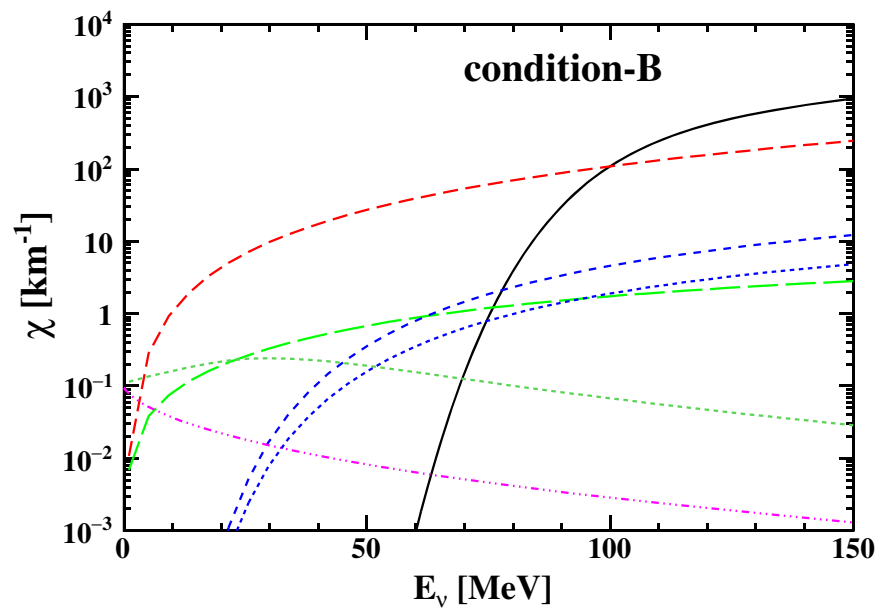

FIG. 5. Comparison of $\nu_{\mu}$ opacities from different weak muonic processes. The left and right plots are for conditions A and B, respectively. We also include $\bar{\nu}_{e}+e^{-} \rightarrow \mu^{-}+\bar{\nu}_{\mu}$ to show its relevance in producing $\mu^{-}$. 

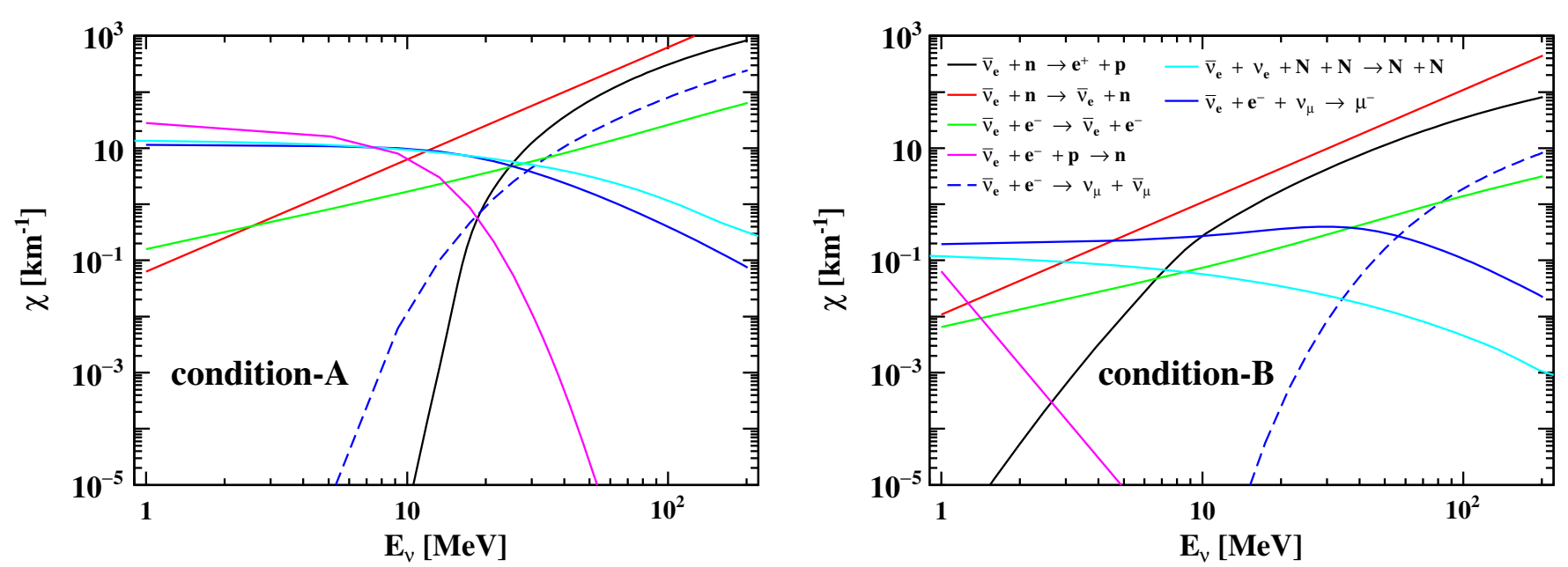

FIG. 6. Comparison of $\bar{\nu}_{e}$ opacities from different weak processes. The left and right plots are for conditions A and B, respectively.

are even higher than those of the $\mathrm{NC}$ ones at high $E_{\nu}$, making $\nu_{\mu}$ more easily trapped. In addition to these semileptonic processes, inverse muon decay can be important opacity sources for low energy $\nu_{\mu}$. We also include neutrino pair annihilation on two nucleons (also called inverse bremsstrahlung) based on the $T$-matrix formalism from Ref. [22], and find that it can contribute with a comparable amount as inverse muon decay at low $E_{\nu}$. Scattering or absorption of $\nu_{\mu}$ on electrons is relevant at intermediate $E_{\nu}$, and can play a role in determining the neutrino spectra via efficient energy exchange. Scattering with $e^{+}$and $\mu^{-}$also contribute to $\nu_{\mu}$ opacities and can be included similarly. Besides, $\nu_{\mu} \bar{\nu}_{\mu}$ pair annihilation to $e^{ \pm}$or other neutrino pairs and $\nu_{\mu}-\nu_{\alpha}$ scattering are subdominant for most of the relevant conditions and are not shown. ${ }^{4}$ It should be mentioned that at high temperature and close to saturation density $\nu_{\mu}+\pi^{-} \rightarrow \mu^{-}$is kinematically allowed due a strong attractive potential for pions, and could dominate the opacity for $E_{\nu} \lesssim 20 \mathrm{MeV}$ [38].

\section{Inverse neutron decay and inverse muon decay as opacity source for $\bar{\nu}_{e}$}

As mentioned above, all the formulas presented in this work can apply equally to $\nu_{e}$ and $\bar{\nu}_{e}$. The opacity of $\bar{\nu}_{e}$ is dominated by $\mathrm{NC}$ scattering on neutrons followed by $\mathrm{CC}$ absorption on protons. With increasing density, the energy difference between neutrons and protons increases and hence the absorption rate at low $E_{\bar{\nu}_{e}}$ is strongly suppressed. On the other hand, inverse processes including inverse neutron decay, inverse muon decay as well as inverse bremsstrahlung could be important opacity sources. In Fig. 6, we compare opacities of $\bar{\nu}_{e}$ from all relevant processes. At condition A,

\footnotetext{
${ }^{4}$ Note that the inverse process, i.e, $e^{ \pm}$annihilation to neutrino pair, is important for the thermal production of neutrinos, especially for the heavy flavors.
}

all the inverse processes dominate the $\bar{\nu}_{e}$ opacity at low energies below $\sim 10 \mathrm{MeV}$. At condition $\mathrm{B}$, the relevance of these inverse processes become less significant as the phase space shrinks with temperature. The phase space for inverse neutron decay rate is further suppressed by a smaller $\Delta U_{n p}$. The role of inverse neutron decay on supernova neutrinos has recently been studied [33]. Note that all the corrections in the hadronic current, including weak magnetism, pseudoscalar term, and form factor effect, can be neglected for inverse neutron decay as they are only important at high $E_{\nu}$.

\section{SUBROUTINES AND TABLES FOR NEUTRINO ABSORPTION ON NUCLEONS}

We provide subroutines for calculating CC neutrinonucleon opacities for CCSN simulations at https://git.gsi .de/nucastro_archive/supernovamuonicrates. Specifically, two different schemes discussed in this work, one invoking a 2D integral and the other using a 4D Monte Carlo integration, have been applied to calculate the opacities. For both subroutines, interaction terms including weak magnetism, pseudoscalar term as well as form factor effects can be included or turned off. As mentioned, the 2D subroutine only treats the leading-order terms of the form factor effects, and may give wrong opacities for $E_{\nu}$ above $\sim 100 \mathrm{MeV}$, while the 4D subroutine provides an exact description. In principle, both subroutines can be called in-flight for neutrino transport in CCSN simulations. The 2D subroutine accounting for weak magnetism using 32-grid-point Gauss quadratures for each dimension has been shown to be efficient and stable in symmetric CCSN simulations [33]. ${ }^{5}$ The 4D subroutine, on the other hand, could be inefficient. Using less grid points or Monte Carlo points may provide ways to trade-off accuracy for

\footnotetext{
${ }^{5}$ We have checked that the $2 \mathrm{D}$ subroutine using 32 grids for each dimension are enough to reach an accuracy of $5 \%$.
} 
computational efficiency for both subroutines. Another possibility is to first tabulate neutrino opacities consistent with the nuclear EoS.

\section{SUMMARY}

In this work we derive analytical formulas for $\mathrm{CC}$ reactions involving $\nu_{\mu} / \bar{\nu}_{\mu}$ or $\mu^{ \pm}$with full relativistic kinematics in the mean field approximation. For the semileptonic reactions, we present an accurate treatment of the hadronic weak current including weak magnetism, pseudoscalar term, and form factor effects. Although weak magnetism increases $\nu_{\mu}$ reaction rate, the pseudoscalar term and the nucleon form factors tend to reduce it. For antineutrinos, on the other hand, all these corrections suppress the opacities. The formulas presented in this work can also be applied to $\nu_{e}\left(\bar{\nu}_{e}\right)$ reactions. Although the pseudoscalar term has negligible effect, the nucleon form factors can reduce the $\nu_{e}$ rate and cancel the enhancement effect due to weak magnetism as for $\nu_{\mu}$.

The muonic semileptonic process dominates the $\nu_{\mu}$ opacity at $E_{\nu} \gtrsim 60-100 \mathrm{MeV}$ and plays essential roles in $\mu^{-}$production and muonization shortly after core bounce. Purely leptonic processes involving $\nu_{\mu}$ or $\mu^{-}$have also been studied and compared to the semileptonic ones. Scattering and absorption on electrons are major inelastic channels for low and intermediate energy $\nu_{\mu}$. At densities higher than $\sim 10^{13} \mathrm{~g} \mathrm{~cm}^{-3}$ as explored in this work, inverse muon decay becomes the dominant inelastic reaction at $E_{\nu} \lesssim 20 \mathrm{MeV}$. As density further increases, inverse muon decay as well as inverse bremsstrahlung can contribute predominantly to $\nu_{\mu}$ opacities of energy below $\sim 10 \mathrm{MeV}$.

The opacity for $\bar{\nu}_{e}$ absorption on proton is suppressed due to a relatively low proton density or a larger energy difference between neutrons and protons at high densities. Similarly to $\nu_{\mu}$, inverse muon decay and inverse bremsstrahlung are also shown to be important reactions for low energy $\bar{\nu}_{e}$ at densities higher than $\sim 10^{13} \mathrm{~g} \mathrm{~cm}^{-3}$. As the energy difference between neutrons and protons in nuclear medium increases, inverse neutron decay, with a larger phase space available, also acts as an important opacity source for low energy $\bar{\nu}_{e}$.

To study the impact of different weak processes, especially muonic reactions, we have considered two specific conditions encountered in PNS $\sim 0.4 \mathrm{~s}$ post core bounce, with densities $\gtrsim 10^{13} \mathrm{~g} \mathrm{~cm}^{-3}$. Although they may not contribute directly in the decoupling region with low densities, the early muonization and the sequent transport of neutrinos could have a non-negligible impact. In particular, considering that low energy neutrinos decouple in deeper regions, these inverse processes could be more interesting. As the PNS contracts and cools down, the effective neutrinosphere will move to high density regions, making the new reactions studied in our work more relevant. In a companion paper of us to be submitted, the impact of these weak muonic processes on the evolution of PNS and neutrino emissions has also been quantified. We also provide subroutines for computing the CC neutrino-nucleon reaction rates, which can be applied in CCSN simulations.

\section{ACKNOWLEDGMENTS}

We thank Robert Bollig and Thomas Janka for providing profiles of a 2D supernova model from Ref. [34] for this work. G. G. acknowledges support from the Academia Sinica by Grant No. AS-CDA-109-M11. G. M. P. acknowledges the support of the Deutsche Forschungsgemeinschaft (DFG, German Research Foundation)-Project-ID 279384907-SFB 1245 "Nuclei: From Fundamental Interactions to Structure and Stars". T. F. acknowledges support from the Polish National Science Center (NCN) under Grants No. 2016/23/B/ST2/00720 and No. 2019/33/ B/ST9/03059.

\section{APPENDIX A: AMPLITUDES AND COEFFICIENTS FOR LEPTONIC REACTIONS}

For all the leptonic processes (a)-(e) listed in Table I, the coefficients $\lambda_{i}$ in Eq. (2) for neutrino scattering on charged leptons and $\lambda_{A i}$ in Eq. (14) for inverse muon decay are given by:

$$
\begin{aligned}
& \lambda_{1}^{(a)}=16 G_{\mathrm{F}}^{2}\left(1-4 s_{W}^{2}+4 s_{W}^{4}\right), \quad \lambda_{2}^{(a)}=64 G_{\mathrm{F}}^{2} s_{W}^{4}, \quad \lambda_{3}^{(a)}=16 G_{\mathrm{F}}^{2} m_{e}^{2}\left(2 s_{W}^{2}-4 s_{W}^{4}\right), \\
& \lambda_{1}^{(b)}=64 G_{\mathrm{F}}^{2} s_{W}^{4}, \quad \lambda_{2}^{(b)}=16 G_{\mathrm{F}}^{2}\left(1-4 s_{W}^{2}+4 s_{W}^{4}\right), \quad \lambda_{3}^{(b)}=16 G_{\mathrm{F}}^{2} m_{e}^{2}\left(2 s_{W}^{2}-4 s_{W}^{4}\right), \\
& \lambda_{1}^{(c)}=64 G_{\mathrm{F}}^{2}, \quad \lambda_{2}^{(c)}=0, \quad \lambda_{3}^{(c)}=0, \\
& \lambda_{1}^{(d)}=0, \quad \lambda_{2}^{(d)}=64 G_{\mathrm{F}}^{2}, \quad \lambda_{3}^{(d)}=0, \\
& \lambda_{A 1}^{(e)}=64 G_{\mathrm{F}}^{2}, \quad \lambda_{A 2}^{(e)}=0, \quad \lambda_{A 3}^{(e)}=0 .
\end{aligned}
$$


The coefficients $A_{i}, B_{i}$, and $C_{i}$ in Eq. (3) are universal for all scattering processes and are given by

$$
\begin{gathered}
A_{1}=E_{1} E_{3}(1-\mu)^{2}\left[E_{1}^{2}+E_{1} E_{3}(3+\mu)+E_{3}^{2}\right], \\
B_{1}=E_{1}^{2} E_{3}(1-\mu)^{2}\left[2 E_{1}^{2}+E_{1} E_{3}(3-\mu)-E_{3}^{2}(1+3 \mu)\right]+Q(1-\mu)\left[E_{1}^{3}+E_{1}^{2} E_{3}(2+\mu)-E_{1} E_{3}^{2}(2+\mu)-E_{3}^{3}\right], \\
C_{1}=E_{1}^{3} E_{3}(1-\mu)^{2}\left[E_{1}^{2}-2 E_{1} E_{3} \mu+E_{3}^{2}\left(-\frac{1}{2}+\frac{3}{2} \mu^{2}\right)\right]+Q E_{1}(1-\mu)\left[E_{1}^{3}-E_{1}^{2} E_{3} \mu+E_{1} E_{3}^{2}\left(-2+\mu^{2}\right)+E_{3}^{3} \mu\right] \\
+Q^{2}\left[E_{1}^{2} \mu-E_{1} E_{3}\left(\frac{3}{2}+\frac{1}{2} \mu^{2}\right)+E_{3}^{2} \mu\right]+\frac{1}{2} E_{1} E_{3}\left(1-\mu^{2}\right) \Delta^{2} m_{2}^{2}, \\
\left\{A_{2}, B_{2}, C_{2}\right\}=\left.\left\{A_{1}, B_{1}, C_{1}\right\}\right|_{E_{1} \leftrightarrow-E_{3}}, \\
A_{3}=B_{3}=0, \quad C_{3}=(1-\mu) \Delta^{4},
\end{gathered}
$$

where $\Delta=\sqrt{E_{1}^{2}-2 E_{1} E_{3} \mu+E_{3}^{2}}, Q=\left(m_{4}^{2}-m_{2}^{2}\right) / 2$, and the coefficients $A_{A i}, B_{A i}, C_{A i}$ for inverse decay in Eq. (16) are simply

$$
\left\{A_{A i}, B_{A i}, C_{A i}\right\}=\left.\left\{A_{i}, B_{i}, C_{i}\right\}\right|_{E_{3} \rightarrow-E_{3}} .
$$

\section{APPENDIX B: MATRIX ELEMENTS AND COEFFICIENTS FOR SEMILEPTONIC REACTIONS}

\section{Matrix elements}

Matrix elements of neutrino absorption on nucleon, $\nu_{l}\left(p_{1}\right)+N_{2}\left(p_{2}\right) \rightarrow l^{-}\left(p_{3}\right)+N_{4}\left(p_{4}\right)$, as shown in Eq. (29), are

$$
\begin{gathered}
\left\langle|\mathcal{M}|^{2}\right\rangle_{V V}=16 G^{2} G_{V}^{2}\left[\left(p_{1} \cdot p_{2}^{*}\right)\left(p_{3} \cdot p_{4}^{*}\right)+\left(p_{1} \cdot p_{4}^{*}\right)\left(p_{3} \cdot p_{2}^{*}\right)-m_{2}^{*} m_{4}^{*}\left(p_{1} \cdot p_{3}\right)\right], \\
\left\langle|\mathcal{M}|^{2}\right\rangle_{V A}=32 G^{2} G_{V} G_{A}\left[\left(p_{1} \cdot p_{2}^{*}\right)\left(p_{3} \cdot p_{4}^{*}\right)-\left(p_{1} \cdot p_{4}^{*}\right)\left(p_{3} \cdot p_{2}^{*}\right)\right], \\
|\mathcal{M}|_{A A}^{2}=16 G^{2} G_{A}^{2}\left[\left(p_{1} \cdot p_{2}^{*}\right)\left(p_{3} \cdot p_{4}^{*}\right)+\left(p_{1} \cdot p_{4}^{*}\right)\left(p_{3} \cdot p_{2}^{*}\right)+m_{2}^{*} m_{4}^{*}\left(p_{1} \cdot p_{3}\right)\right], \\
\left\langle|\mathcal{M}|^{2}\right\rangle_{V F}=\frac{16 G^{2} G_{V} F_{2}}{2 M_{N}}\left\{\left[\left(p_{1} \cdot p_{2}^{*}\right) m_{4}^{*}-\left(p_{1} \cdot p_{4}^{*}\right) m_{2}^{*}\right]\left(p_{3} \cdot q^{*}\right)+\left[\left(p_{3} \cdot p_{2}^{*}\right) m_{4}^{*}-\left(p_{3} \cdot p_{4}^{*}\right) m_{2}^{*}\right]\left(p_{1} \cdot q^{*}\right)\right. \\
\left.+\left[\left(q^{*} \cdot p_{2}^{*}\right) m_{4}^{*}-\left(q^{*} \cdot p_{4}^{*}\right) m_{2}^{*}\right]\left(p_{1} \cdot p_{3}\right)\right\} \\
\left\langle|\mathcal{M}|^{2}\right\rangle_{A F}=\frac{16 G^{2} G_{A} F_{2}}{M_{N}}\left\{\left[\left(p_{1} \cdot p_{2}^{*}\right) m_{4}^{*}+\left(p_{1} \cdot p_{4}^{*}\right) m_{2}^{*}\right]\left(p_{3} \cdot q^{*}\right)-\left[\left(p_{3} \cdot p_{2}^{*}\right) m_{4}^{*}+\left(p_{3} \cdot p_{4}^{*}\right) m_{2}^{*}\right]\left(p_{1} \cdot q^{*}\right)\right\}, \\
\left\langle|\mathcal{M}|^{2}\right\rangle_{F F}=\frac{16 G^{2} F_{2}^{2}}{8 M_{N}^{2}}\left\{2\left[\left(p_{3} \cdot p_{2}^{*}\right)\left(p_{4}^{*} \cdot q^{*}\right)+\left(p_{3} \cdot p_{4}^{*}\right)\left(p_{2}^{*} \cdot q^{*}\right)\right]\left(p_{1} \cdot q^{*}\right)+2\left[\left(p_{1} \cdot p_{2}^{*}\right)\left(p_{4}^{*} \cdot q^{*}\right)+\left(p_{1} \cdot p_{4}^{*}\right)\left(p_{2}^{*} \cdot q^{*}\right)\right]\left(p_{3} \cdot q^{*}\right)\right. \\
-2\left(p_{1} \cdot q^{*}\right)\left(p_{3} \cdot q^{*}\right)\left(p_{2}^{*} \cdot p_{4}^{*}\right)+q^{* 2}\left[\left(p_{1} \cdot p_{3}\right)\left(p_{2}^{*} \cdot p_{4}^{*}\right)-2\left(p_{1} \cdot p_{2}^{*}\right)\left(p_{3} \cdot p_{4}^{*}\right)-2\left(p_{1} \cdot p_{4}^{*}\right)\left(p_{3} \cdot p_{2}^{*}\right)\right] \\
\left.-m_{2}^{*} m_{4}^{*}\left[\left(p_{1} \cdot p_{3}\right) q^{* 2}+2\left(p_{1} \cdot q^{*}\right)\left(p_{3} \cdot q^{*}\right)\right]\right\} \\
\left\langle|\mathcal{M}|^{2}\right\rangle_{A P}=\frac{16 G^{2} G_{A} G_{P}}{M_{N}}\left\{\left(p_{1} \cdot q^{*}\right)\left[\left(p_{3} \cdot p_{2}^{*}\right) m_{4}^{*}-\left(p_{3} \cdot p_{4}^{*}\right) m_{2}^{*}\right]+\left(p_{3} \cdot q^{*}\right)\left[\left(p_{1} \cdot p_{2}^{*}\right) m_{4}^{*}-\left(p_{1} \cdot p_{4}^{*}\right) m_{2}^{*}\right]\right. \\
\left.+\left(p_{1} \cdot p_{3}\right)\left[\left(q^{*} \cdot p_{4}^{*}\right) m_{2}^{*}-\left(q^{*} \cdot p_{2}^{*}\right) m_{4}^{*}\right]\right\} \\
\left\langle|\mathcal{M}|^{2}\right\rangle_{P P}=\frac{16 G^{2} G_{P}^{2}}{2 M_{N}^{2}}\left[2\left(p_{1} \cdot q^{*}\right)\left(p_{3} \cdot q^{*}\right)-q^{* 2}\left(p_{1} \cdot p_{3}\right)\right]\left[\left(p_{2}^{*} \cdot p_{4}^{*}\right)-m_{2}^{*} m_{4}^{*}\right],
\end{gathered}
$$


where $G=G_{\mathrm{F}} V_{u d}$ with $G_{\mathrm{F}}$ the Fermi coupling constant and $V_{u d}$ the up-down entry of the Cabibbo-KobayashiMaskawa matrix.

\section{2. $I_{\mathcal{X}}$ and the coefficients $\mathcal{X}$ in Eq. (33)}

We take $I_{\mathcal{A}}$ for example, and demonstrate how the angular integration can be solved. From the definitions in Eqs. (31) and (34), $I_{\mathcal{A}}$ is given by

$$
\begin{aligned}
I_{\mathcal{A}}= & \frac{\bar{p}_{1} \bar{p}_{2} \bar{p}_{3} \bar{p}_{4}}{4 \pi^{2}} \int d \boldsymbol{\Omega}_{2} d \boldsymbol{\Omega}_{3} d \boldsymbol{\Omega}_{4}\left(p_{1} \cdot p_{2}^{*}\right)\left(p_{3} \cdot p_{4}^{*}\right) \\
& \times \delta^{(3)}\left(\boldsymbol{p}_{1}+\boldsymbol{p}_{2}-\boldsymbol{p}_{3}-\boldsymbol{p}_{4}\right) .
\end{aligned}
$$

Define $\boldsymbol{p}_{a}=\boldsymbol{p}_{1}+\boldsymbol{p}_{2}$ and $\cos \theta_{2}=x_{2}$ with $\theta_{2}$ the angle between $\boldsymbol{p}_{1}$ and $\boldsymbol{p}_{2}$, we have

$$
\bar{p}_{a}=\sqrt{\bar{p}_{1}^{2}+\bar{p}_{2}^{2}+2 \bar{p}_{1} \bar{p}_{2} x_{2}},
$$

with the notation $\bar{p}_{i}=\left|\boldsymbol{p}_{i}\right|$. The momentum delta function can be expressed as

$$
\begin{aligned}
& \delta^{(3)}\left(\boldsymbol{p}_{1}+\boldsymbol{p}_{2}-\boldsymbol{p}_{3}-\boldsymbol{p}_{4}\right) \\
& \left.=\frac{1}{\bar{p}_{4}^{2}} \delta\left(\bar{p}_{4}-\left|\boldsymbol{p}_{a}-\boldsymbol{p}_{3}\right|\right) \delta^{(2)}\left(\Omega_{4}-\Omega_{\left|\boldsymbol{p}_{a}-\boldsymbol{p}_{3}\right|}\right)\right) .
\end{aligned}
$$

Integrating over $d \Omega_{4}$, we obtain

$$
\begin{aligned}
I_{\mathcal{A}}= & \frac{\bar{p}_{1} \bar{p}_{2} \bar{p}_{3}}{4 \pi^{2} \bar{p}_{4}} \int d \Omega_{2} d \Omega_{3}\left(E_{1} E_{2}^{*}-\boldsymbol{p}_{1} \cdot \boldsymbol{p}_{2}\right)\left(E_{3} E_{4}^{*}-\boldsymbol{p}_{3} \cdot \boldsymbol{p}_{4}\right) \\
& \times \delta\left(\bar{p}_{4}-\left|\boldsymbol{p}_{a}-\boldsymbol{p}_{3}\right|\right) .
\end{aligned}
$$

The angular integral for particle ' 3 ' is given by $d \Omega_{3}=$ $d \phi_{3} d x_{3}$. One has the freedom to define $x_{3}=\cos \theta_{a 3}$, with $\theta_{a 3}$ the angle between $\boldsymbol{p}_{a}$ and $\boldsymbol{p}_{3}$. This allows for a variable substitution in the $\delta$-distribution

$\delta\left(\bar{p}_{4}-\left|\boldsymbol{p}_{a}-\boldsymbol{p}_{3}\right|\right)=\frac{\bar{p}_{4}}{\bar{p}_{a} \bar{p}_{3}} \delta\left(x_{3}-\frac{\bar{p}_{a}^{2}+\bar{p}_{3}^{2}-\bar{p}_{4}^{2}}{2 \bar{p}_{a} \bar{p}_{3}}\right)$.

Now one can rewrite the momentum product $\boldsymbol{p}_{3} \cdot \boldsymbol{p}_{4}$ as

$\boldsymbol{p}_{3} \cdot \boldsymbol{p}_{4}=\boldsymbol{p}_{3} \cdot\left(\boldsymbol{p}_{a}-\boldsymbol{p}_{3}\right)=\bar{p}_{3} \bar{p}_{a} x_{3}-\bar{p}_{3}^{2}=\frac{\bar{p}_{a}^{2}-\bar{p}_{3}^{2}-\bar{p}_{4}^{2}}{2}$.

Similarly it is trivial to show that

$$
\boldsymbol{p}_{1} \cdot \boldsymbol{p}_{2}=\frac{\bar{p}_{a}^{2}-\bar{p}_{1}^{2}-\bar{p}_{2}^{2}}{2}
$$

Performing integration over $\phi_{3}$ and $x_{3}, I_{\mathcal{A}}$ then becomes

$$
\begin{aligned}
I_{\mathcal{A}}= & \frac{\bar{p}_{1} \bar{p}_{2}}{2 \pi} \int d \Omega_{2} \frac{1}{\bar{p}_{a}} \Theta\left(\bar{p}_{3}+\bar{p}_{4}-\bar{p}_{a}\right) \Theta\left(\bar{p}_{a}-\left|\bar{p}_{3}-\bar{p}_{4}\right|\right) \\
& \times\left(E_{1} E_{2}^{*}+\frac{\bar{p}_{1}^{2}+\bar{p}_{2}^{2}-\bar{p}_{a}^{2}}{2}\right)\left(E_{3} E_{4}^{*}+\frac{\bar{p}_{3}^{2}+\bar{p}_{4}^{2}-\bar{p}_{a}^{2}}{2}\right),
\end{aligned}
$$

where the Heaviside functions come from the cosine limits $-1 \leq x_{3} \leq 1$. From Eq. (B3) one can substitute the angular integral over $\Omega_{2}$ by

$$
d \Omega_{2}=d \phi_{2} d x_{2}=d \phi_{2} d \bar{p}_{a} \frac{\bar{p}_{a}}{\bar{p}_{1} \bar{p}_{2}},
$$

then the integral $I_{\mathcal{A}}$ takes the form

$$
\begin{aligned}
I_{\mathcal{A}}= & \int_{p_{a-}}^{p_{a+}} d \bar{p}_{a}\left(E_{1} E_{2}^{*}+\frac{\bar{p}_{1}^{2}+\bar{p}_{2}^{2}-\bar{p}_{a}^{2}}{2}\right) \\
& \times\left(E_{3} E_{4}^{*}+\frac{\bar{p}_{3}^{2}+\bar{p}_{4}^{2}-\bar{p}_{a}^{2}}{2}\right) .
\end{aligned}
$$

The integration limits $p_{a-}$ and $p_{a+}$ arise from the combined constraints on $x_{2}$ and $x_{3}$,

$$
\begin{gathered}
p_{a-}=\max \left\{\left|\bar{p}_{1}-\bar{p}_{2}\right|,\left|\bar{p}_{3}-\bar{p}_{4}\right|\right\}, \\
p_{a+}=\min \left\{\bar{p}_{1}+\bar{p}_{2}, \bar{p}_{3}+\bar{p}_{4}\right\} .
\end{gathered}
$$

Performing the integration over $\bar{p}_{a}, I_{\mathcal{A}}$ becomes

$$
\begin{aligned}
I_{\mathcal{A}}= & \frac{1}{60}\left[3\left(p_{a+}^{5}-p_{a-}^{5}\right)-10(a+b)\left(p_{a+}^{3}-p_{a-}^{3}\right)\right. \\
& \left.+60 a b\left(p_{a+}-p_{a-}\right)\right],
\end{aligned}
$$

where coefficients $a$ and $b$ are given by

$$
a=E_{1} E_{2}^{*}+\frac{\bar{p}_{1}^{2}+\bar{p}_{2}^{2}}{2}, \quad b=E_{3} E_{4}^{*}+\frac{\bar{p}_{3}^{2}+\bar{p}_{4}^{2}}{2} .
$$

Taking similar steps, one can solve the angular integrals for all $I_{\mathcal{X}}$, and obtain

$$
\begin{aligned}
& I_{\mathcal{A}}=\frac{1}{60}\left[3\left(p_{a+}^{5}-p_{a-}^{5}\right)-10(a+b)\left(p_{a+}^{3}-p_{a-}^{3}\right)+60 a b\left(p_{a+}-p_{a-}\right)\right], \\
& I_{\mathcal{B}}=\frac{1}{60}\left[3\left(p_{b+}^{5}-p_{b-}^{5}\right)-10(c+d)\left(p_{b+}^{3}-p_{b-}^{3}\right)+60 c d\left(p_{b+}-p_{b-}\right)\right],
\end{aligned}
$$




$$
\begin{aligned}
& I_{\mathcal{C}}=\left[-\frac{\left(p_{a+}^{7}-p_{a-}^{7}\right)}{112}+\frac{a+\alpha_{1}}{20}\left(p_{a+}^{5}-p_{a-}^{5}\right)-\frac{a^{2}+4 a \alpha_{1}-\alpha_{0}}{12}\left(p_{a+}^{3}-p_{a-}^{3}\right)\right. \\
& \left.+\left(a^{2} \alpha_{1}-a \alpha_{0}\right)\left(p_{a+}-p_{a-}\right)-a^{2} \alpha_{0}\left(p_{a+}^{-1}-p_{a-}^{-1}\right)\right], \\
& I_{\mathcal{D}}=\left[\frac{\left(p_{c+}^{7}-p_{c-}^{7}\right)}{112}+\frac{e+\epsilon_{1}}{20}\left(p_{c+}^{5}-p_{c-}^{5}\right)+\frac{e^{2}+4 e \epsilon_{1}+\epsilon_{0}}{12}\left(p_{c+}^{3}-p_{c-}^{3}\right)\right. \\
& \left.+\left(e^{2} \epsilon_{1}+e \epsilon_{0}\right)\left(p_{c+}-p_{c-}\right)-e^{2} \epsilon_{0}\left(p_{c+}^{-1}-p_{c-}^{-1}\right)\right] \\
& I_{\mathcal{E}}=\frac{1}{60}\left[3\left(p_{a+}^{5}-p_{a-}^{5}\right)-20 a\left(p_{a+}^{3}-p_{a-}^{3}\right)+60 a^{2}\left(p_{a+}-p_{a-}\right)\right], \\
& I_{\mathcal{F}}=\frac{1}{60}\left[3\left(p_{c+}^{5}-p_{c-}^{5}\right)+20 e\left(p_{c+}^{3}-p_{c-}^{3}\right)+60 e^{2}\left(p_{c+}-p_{c-}\right)\right] \\
& I_{\mathcal{H}}=\left[\frac{p_{c+}^{5}-p_{c-}^{5}}{40}+\frac{e+2 \epsilon_{1}}{12}\left(p_{c+}^{3}-p_{c-}^{3}\right)+\frac{2 e \epsilon_{1}+\epsilon_{0}}{2}\left(p_{c+}-p_{c-}\right)-e \epsilon_{0}\left(p_{c+}^{-1}-p_{c-}^{-1}\right)\right], \\
& I_{\mathcal{J}}=\frac{1}{6}\left[-\left(p_{a+}^{3}-p_{a-}^{3}\right)+6 a\left(p_{a+}-p_{a-}\right)\right] \\
& I_{\mathcal{K}}=\frac{1}{6}\left[\left(p_{c+}^{3}-p_{c-}^{3}\right)+6 e\left(p_{c+}-p_{c-}\right)\right] \\
& I_{\mathcal{L}}=p_{a+}-p_{a-}, \\
& I_{\mathcal{L}}^{A P}= \begin{cases}\frac{1}{\sqrt{Z_{P}}}\left[\arctan \left(\frac{p_{c+}}{\sqrt{Z_{P}}}\right)-\arctan \left(\frac{p_{c-}}{\sqrt{Z_{P}}}\right)\right], & \text { if } Z_{P}>0 \\
\log \left(\left|\frac{1+\eta_{P}}{1-\eta_{P}}\right|\right) /\left(2 \sqrt{\left|Z_{P}\right|}\right), & \text { if } Z_{P} \leq 0\end{cases} \\
& I_{\mathcal{K}}^{A P}=\frac{1}{2}\left[\left(p_{c+}-p_{c-}\right)+\left(2 e-Z_{P}\right) I_{\mathcal{L}}^{A P}\right] \\
& I_{\mathcal{J}}^{A P}=\epsilon_{1} I_{\mathcal{L}}^{A P}+\frac{1}{4}\left[\left(p_{c+}-p_{c-}\right)-Z_{P} I_{\mathcal{L}}^{A P}\right]-\frac{\epsilon_{0}}{Z_{P}}\left(\frac{1}{p_{c+}}-\frac{1}{p_{c-}}+I_{\mathcal{L}}^{A P}\right) \\
& =\left(\epsilon_{1}-\frac{\epsilon_{0}}{Z_{P}}-\frac{Z_{P}}{4}\right) I_{\mathcal{L}}^{A P}+\left[\frac{1}{4}\left(p_{c+}-p_{c-}\right)-\frac{\epsilon_{0}}{Z_{P}}\left(\frac{1}{p_{c+}}-\frac{1}{p_{c-}}\right)\right], \\
& I_{\mathcal{L}}^{P P}=\frac{1}{2 Z_{P}}\left(\frac{p_{c+}}{p_{c+}^{2}+Z_{P}}-\frac{p_{c-}}{p_{c-}^{2}+Z_{P}}+I_{\mathcal{L}}^{A P}\right), \\
& I_{\mathcal{K}}^{P P}=\left(e-\frac{Z_{P}}{2}\right) I_{\mathcal{L}}^{P P}+\frac{I_{\mathcal{L}}^{A P}}{2}, \\
& I_{\mathcal{F}}^{P P}=e^{2} I_{\mathcal{L}}^{P P}+e\left(-Z_{P} I_{\mathcal{L}}^{P P}+I_{\mathcal{L}}^{A P}\right)+\frac{1}{4}\left[Z_{P}^{2} I_{\mathcal{L}}^{P P}-2 Z_{P} I_{\mathcal{L}}^{A P}+\left(p_{c+}-p_{c-}\right)\right] \\
& =\left(e-\frac{Z_{P}}{2}\right)^{2} I_{\mathcal{L}}^{P P}+\left(e-\frac{Z_{P}}{2}\right) I_{\mathcal{L}}^{A P}+\frac{p_{c+}-p_{c-}}{4},
\end{aligned}
$$

with 


$$
\begin{aligned}
& a=E_{1} E_{2}^{*}+\frac{\bar{p}_{1}^{2}+\bar{p}_{2}^{2}}{2}, \quad b=E_{3} E_{4}^{*}+\frac{\bar{p}_{3}^{2}+\bar{p}_{4}^{2}}{2}, \\
& c=-E_{1} E_{4}^{*}+\frac{\bar{p}_{1}^{2}+\bar{p}_{4}^{2}}{2}, \quad d=-E_{3} E_{2}^{*}+\frac{\bar{p}_{2}^{2}+\bar{p}_{3}^{2}}{2}, \quad e=E_{1} E_{3}-\frac{\bar{p}_{1}^{2}+\bar{p}_{3}^{2}}{2}, \\
& \alpha_{0}=\frac{1}{4}\left(\bar{p}_{1}^{2}-\bar{p}_{2}^{2}\right)\left(\bar{p}_{4}^{2}-\bar{p}_{3}^{2}\right), \quad \alpha_{1}=E_{1} E_{3}-\frac{1}{4}\left(\bar{p}_{1}^{2}-\bar{p}_{2}^{2}+\bar{p}_{3}^{2}-\bar{p}_{4}^{2}\right), \\
& \epsilon_{0}=\frac{1}{4}\left(\bar{p}_{1}^{2}-\bar{p}_{3}^{2}\right)\left(\bar{p}_{2}^{2}-\bar{p}_{4}^{2}\right), \quad \epsilon_{1}=E_{1} E_{2}^{*}+\frac{1}{4}\left(\bar{p}_{1}^{2}+\bar{p}_{2}^{2}-\bar{p}_{3}^{2}-\bar{p}_{4}^{2}\right), \\
& Z_{P}=m_{\pi}^{2}-m_{3}^{2}+2 E_{1} E_{3}-\bar{p}_{1}^{2}-\bar{p}_{3}^{2}-2 \Delta U\left(E_{1}-E_{3}\right)-\Delta U^{2}, \quad \eta_{P}=\sqrt{\left|Z_{P}\right|} \frac{p_{c+}-p_{c-}}{p_{c+} p_{c-}+Z_{P}},
\end{aligned}
$$

and

$$
\begin{array}{ll}
p_{a-}=\max \left\{\left|\bar{p}_{1}-\bar{p}_{2}\right|,\left|\bar{p}_{3}-\bar{p}_{4}\right|\right\}, & p_{a+}=\min \left\{\bar{p}_{1}+\bar{p}_{2}, \bar{p}_{3}+\bar{p}_{4}\right\} \\
p_{b-}=\max \left\{\left|\bar{p}_{1}-\bar{p}_{4}\right|,\left|\bar{p}_{2}-\bar{p}_{3}\right|\right\}, & p_{b+}=\min \left\{\bar{p}_{1}+\bar{p}_{4}, \bar{p}_{2}+\bar{p}_{3}\right\}, \\
p_{c-}=\max \left\{\left|\bar{p}_{1}-\bar{p}_{3}\right|,\left|\bar{p}_{2}-\bar{p}_{4}\right|\right\}, & p_{c+}=\min \left\{\bar{p}_{1}+\bar{p}_{3}, \bar{p}_{2}+\bar{p}_{4}\right\} .
\end{array}
$$

The coefficients $\mathcal{X}, \mathcal{X}^{A P, P P}$ in Eq. (33) are

$$
\begin{aligned}
\mathcal{A}= & \left(g_{V}+g_{A}\right)^{2}+2 g_{A} F_{2} \frac{m_{2}^{*}}{m_{N}}\left(1-\frac{\Delta m^{*}}{2 m_{2}^{*}}\right), \quad \mathcal{B}=\left(g_{V}-g_{A}\right)^{2}-2 g_{A} F_{2} \frac{m_{2}^{*}}{m_{N}}\left(1-\frac{\Delta m^{*}}{2 m_{2}^{*}}\right), \\
\mathcal{C}= & \frac{F_{2}^{2}}{m_{N}^{2}}, \quad \mathcal{D}=-\frac{F_{2}^{2}}{m_{N}^{2}}, \quad \mathcal{E}=-\frac{F_{2}^{2}}{2 m_{N}^{2}}\left[m_{3}^{2}-2 \Delta U\left(E_{3}-E_{1}\right)+\Delta U^{2}\right], \\
\mathcal{F}= & g_{V} F_{2} \frac{m_{2}^{*}}{m_{N}}\left(2-\frac{\Delta m_{*}}{m_{2}^{*}}\right)+\frac{F_{2}^{2}}{2 m_{N}^{2}}\left[m_{2}^{*} m_{4}^{*}-Q_{24}+\frac{m_{3}^{2}}{4}-\Delta U\left(E_{1}+E_{2}^{*}\right)-\frac{\Delta U^{2}}{4}\right], \\
\mathcal{H}= & \frac{F_{2}^{2}}{2 m_{N}^{2}}\left[2 Q_{24}+m_{3}^{2}+\Delta U\left(3 E_{1}-E_{3}+2 E_{4}^{*}\right)\right], \quad \mathcal{J}=g_{V} F_{2} \frac{\Delta m^{*}}{2 m_{N}}\left[m_{3}^{2}-\Delta U\left(E_{1}+E_{3}\right)\right]+\frac{F_{2}^{2}}{2 m_{N}^{2}} \mathcal{J}_{F F}, \\
\mathcal{K}= & \left(g_{A}^{2}-g_{V}^{2}\right) m_{2}^{*} m_{4}^{*}+g_{V} F_{2} \frac{m_{2}^{*}}{2 m_{N}}\left\{-3 m_{3}^{2}+4 \Delta U\left(E_{3}-E_{1}\right)-\Delta U^{2}+\frac{\Delta m^{*}}{m_{2}^{*}}\left[2 Q_{24}+m_{3}^{2}+\Delta U\left(2 E_{1}-E_{3}+E_{4}^{*}\right)\right]\right\} \\
& +\frac{F_{2}^{2}}{2 m_{N}^{2}} \mathcal{K}_{F F}, \\
\mathcal{L}= & g_{v} F_{2} \frac{m_{2}^{*}}{m_{N}} \Delta U E_{1}\left[m_{3}^{2}-\Delta U E_{3}+\frac{\Delta m^{*}}{2 m_{2}^{*}}\left(-Q_{24}-\frac{m_{3}^{2}}{2}-\Delta U E_{4}^{*}+\frac{\Delta U^{2}}{2}\right)\right]+\frac{F_{2}^{2}}{2 m_{N}^{2}} \mathcal{L}_{F F}, \\
\mathcal{F}^{P P}= & 2 m_{N}^{2} g_{A}^{2}\left(m_{3}^{2}-\Delta U^{2}\right), \quad \mathcal{J}^{A P}=2 m_{N} g_{A}^{2} \Delta m^{*}\left[m_{3}^{2}-\Delta U\left(E_{1}+E_{3}\right)\right], \\
\mathcal{K}^{A P}= & 2 g_{A}^{2} m_{N}\left[m_{2}^{*}\left(\Delta U^{2}-m_{3}^{2}\right)+\Delta m^{*} \Delta U\left(E_{1}+E_{2}^{*}\right)\right], \\
\mathcal{K}^{P P}= & 2 g_{A}^{2} m_{N}^{2}\left[\frac{m_{3}^{2}}{2}\left(-m_{3}^{2}+\Delta m^{* 2}\right)+\Delta U m_{3}^{2}\left(E_{3}-3 E_{1}\right)+\Delta U^{2}\left(2 E_{1} E_{3}-\frac{\Delta m^{* 2}}{2}\right)+\Delta U^{3}\left(E_{4}^{*}-E_{2}^{*}\right)-\frac{\Delta U^{4}}{2}\right], \\
\mathcal{L}^{A P}= & 2 g_{A}^{2} m_{N} \Delta U E_{1}\left[2 m_{2}^{*} m_{3}^{2}-\Delta m^{*}\left(Q_{24}+\frac{m_{3}^{2}}{2}\right)-\Delta U\left(2 E_{3} m_{2}^{*}+E_{4}^{*} \Delta m^{*}\right)+\frac{\Delta m^{*}}{2} \Delta U^{2}\right], \\
\mathcal{L}^{P P}= & 2 g_{A}^{2} m_{N}^{2} \Delta U E_{1}\left\{m_{3}^{2}\left(m_{3}^{2}-\Delta m^{* 2}\right)+\Delta U\left[-m_{3}^{2}\left(3 E_{3}-2 E_{1}\right)+\Delta m^{* 2} E_{3}\right]+\Delta U^{2}\left[m_{3}^{2}+2 E_{3}\left(E_{3}-E_{1}\right)\right]-E_{3} \Delta U^{3}\right\},
\end{aligned}
$$

where 


$$
\begin{aligned}
\mathcal{J}_{F F}= & \Delta U\left\{-m_{3}^{2}\left(E_{1}+\frac{E_{2}^{*}+E_{4}^{*}}{2}\right)+Q_{24}\left(E_{3}-3 E_{1}\right)+\frac{\Delta U}{2}\left[E_{4}^{*}\left(3 E_{3}-5 E_{1}\right)+E_{2}^{*}\left(E_{3}+E_{1}\right)+E_{3}^{2}-E_{1}^{2}-2 Q_{24}\right]\right. \\
& \left.+\Delta U^{2}\left(E_{1}-E_{3}-E_{4}^{*}\right)+\frac{\Delta U^{3}}{2}\right\}, \\
\mathcal{K}_{F F}= & -\left(m_{2}^{*}+3 m_{4}^{*}\right) m_{2}^{*} \frac{m_{3}^{2}}{4}+Q_{24}^{2}+Q_{24} \frac{m_{3}^{2}}{4}-\frac{m_{3}^{4}}{8} \\
& +\Delta U\left[\frac{Q_{24}}{2}\left(3 E_{1}-E_{2}^{*}+E_{3}+3 E_{4}^{*}\right)+\frac{m_{3}^{2}}{4}\left(2 E_{2}^{*}+E_{3}+E_{1}\right)+m_{2}^{*} m_{4}^{*}\left(E_{3}-E_{1}\right)\right] \\
& +\Delta U^{2}\left[\frac{1}{4}\left(m_{2}^{* 2}-m_{2}^{*} m_{4}^{*}-3 Q_{24}\right)+\frac{E_{4}^{*}}{2}\left(E_{3}+2 E_{1}-E_{2}^{*}+E_{4}^{*}\right)+E_{2}^{*}\left(\frac{1}{2} E_{1}-E_{3}\right)+\frac{E_{1}^{2}}{2}\right] \\
+ & \frac{\Delta U^{3}}{4}\left(-E_{1}+2 E_{2}^{*}-E_{3}-2 E_{4}^{*}\right)+\frac{\Delta U^{4}}{8}, \\
= & \frac{\Delta U E_{1}}{4}\left\{m_{3}^{2}\left(m_{2}^{*}+m_{4}^{*}\right)^{2}-4 Q_{24}^{2}+\Delta U\left[-m_{3}^{2}\left(E_{2}^{*}+E_{4}^{*}+E_{1}\right)-2 E_{3}\left(m_{2}^{* 2}+m_{2}^{*} m_{4}^{*}\right)+2 Q_{24}\left(E_{2}^{*}-3 E_{4}^{*}-E_{1}\right)\right]\right. \\
& \left.+2 \Delta U^{2}\left[E_{2}^{*} E_{3}+E_{4}^{*}\left(E_{2}^{*}-E_{4}^{*}-E_{1}\right)+Q_{24}\right]+\Delta U^{3}\left[-E_{2}^{*}+E_{4}^{*}+E_{1}\right]\right\},
\end{aligned}
$$

with $\quad \Delta U=U_{2}-U_{4}, \quad \Delta m^{*}=m_{2}^{*}-m_{4}^{*}, \quad$ and $\quad Q_{24}=$ $\left(m_{2}^{* 2}-m_{4}^{* 2}\right) / 2$.

\section{APPENDIX C: 4D INTEGRALS AND PHYSICALLY ALLOWED REGION}

The 2D integrals in this work are done straightforwardly via the Gauss-Legendre quadrature in each dimension. In this subsection, we will show the details of computing the opacities from the 4D integrals. We need to determine the boundaries of the integration variables that are kinematically allowed by energy-momentum conservation.

\section{Bounds for neutrino absorption or scattering}

The discussions below can apply equally to both neutrino scattering and absorption on nucleons and leptons. We take neutrino absorption on nucleons, $\nu_{1}+N_{2} \rightarrow l_{3}+N_{4}$, for demonstration. It can be easily seen, for example, by taking $N_{2,4} \rightarrow l_{2,4}$ and $l_{3} \rightarrow \nu_{3}$, the results for neutrino scattering on leptons are obtained.

For neutrino absorption on nucleons, we set $\boldsymbol{q}=\boldsymbol{p}_{4}-$ $\boldsymbol{p}_{2}=\boldsymbol{p}_{1}-\boldsymbol{p}_{3}$ along the $z$-axis, and choose $E_{2},|\boldsymbol{q}|, \cos \theta_{q 2}$ and $\phi_{q 2}$ as the integration variables, where $\theta_{q 2}$ and $\phi_{q 2}$ are the pole and azimuthal angles of $\boldsymbol{p}_{2}$ with respect to $\boldsymbol{q}$ or the $z$-axis. Once the four variables are fixed, the four-momenta of all the particles can be determined as follows: $\boldsymbol{p}_{2}$ is fixed when $E_{2}, \theta_{q 2}$ and $\phi_{q 2}$ are known; furthermore, $\boldsymbol{p}_{4}$ and then $q^{0}=E_{4}-E_{2}$ can be determined if $\boldsymbol{q}=|\boldsymbol{q}| \hat{z}$ is known. For a given neutrino energy $E_{1}$ with $q^{0}$ and $\boldsymbol{q}$ fixed, $\boldsymbol{p}_{1,3}$ are then uniquely determined.

Since $\quad q_{0}=\sqrt{\left|\boldsymbol{p}_{2}+\boldsymbol{q}\right|^{2}+m_{4}^{* 2}}+U_{4}-E_{2}=E_{1}-$ $\sqrt{\left|\boldsymbol{p}_{1}-\boldsymbol{q}\right|^{2}+m_{3}^{2}}$, the maximal and minimal values of $q_{0}$ can be expressed as

$$
\begin{array}{r}
q_{0}^{H, \text { max }}=\sqrt{\left(\left|\boldsymbol{p}_{2}\right|+|\boldsymbol{q}|\right)^{2}+m_{4}^{* 2}}+U_{4}-E_{2}, \\
q_{0}^{H, \text { min }}=\sqrt{\left(\left|\boldsymbol{p}_{2}\right|-|\boldsymbol{q}|\right)^{2}+m_{4}^{* 2}}+U_{4}-E_{2}, \\
q_{0}^{L, \max }=E_{1}-\sqrt{\left(E_{1}-|\boldsymbol{q}|\right)^{2}+m_{3}^{2}}, \\
q_{0}^{L, \text { min }}=E_{1}-\sqrt{\left(E_{1}+|\boldsymbol{q}|\right)^{2}+m_{3}^{2}} .
\end{array}
$$

For the kinematically allowed values of $\left|\boldsymbol{p}_{2}\right|$ and $|\boldsymbol{q}|$, we should have $q_{0}^{L, \text { max }} \geq q_{0}^{H, \text { min }}$ and meanwhile, $q_{0}^{H, \text { max }} \geq q_{0}^{L, \text { min }}$. From these requirements, we can obtain the upper and lower bounds of the integration variables.

(a) Allowed range of $E_{1}$ and upper/lower bounds of $E_{2}$ or $\left|p_{2}\right|$ :

We note that $q_{0}^{H, \text { max }}$ grows with $|\boldsymbol{q}|$ while $q_{0}^{L, \text { min }}$ decreases, so we can always find the allowed region of $|\boldsymbol{q}|$ for any given value of $\left|\boldsymbol{p}_{2}\right|$ that satisfy the inequality $q_{0}^{H, \text { max }} \geq q_{0}^{L, \text { min }}$. In other words, the allowed range of $\left|\boldsymbol{p}_{2}\right|$ can be determined by requiring that there are solutions of $|\boldsymbol{q}|$ that satisfy $F\left(|\boldsymbol{q}|,\left|\boldsymbol{p}_{2}\right|\right)=q_{0}^{H, \text { min }}-q_{0}^{L, \text { max }} \leq 0$. From

$$
\frac{\partial F\left(|\boldsymbol{q}|,\left|\boldsymbol{p}_{2}\right|\right)}{\partial|\boldsymbol{q}|}=0,
$$

one can find that $F\left(|\boldsymbol{q}|,\left|\boldsymbol{p}_{2}\right|\right)$ reaches its minimum at

$$
|\boldsymbol{q}|_{\min }=\frac{m_{3}\left|\boldsymbol{p}_{2}\right|+m_{4}^{*} E_{1}}{\tilde{m}_{4}^{*}}
$$

with $\tilde{m}_{4}^{*}=m_{4}^{*}+m_{3}$. For $\tilde{m}_{4}^{*}<m_{2}^{*}, F\left(|\boldsymbol{q}|_{\min },\left|\boldsymbol{p}_{2}\right|\right)$ first decreases with $\left|\boldsymbol{p}_{2}\right|$, reaches its minimum at 


$$
\left|\boldsymbol{p}_{2}\right|_{\min }=\frac{E_{1} m_{2}^{*}}{m_{2}^{*}-\tilde{m}_{4}^{*}}
$$

and then increases. For $\tilde{m}_{4}^{*} \geq m_{2}^{*}, F\left(|\boldsymbol{q}|_{\min },\left|\boldsymbol{p}_{2}\right|\right)$ decreases monotonically.

Since the minimum of $F\left(|\boldsymbol{q}|,\left|\boldsymbol{p}_{2}\right|\right)$ should be negative, the allowed values of $E_{1}$ for a nonzero reaction rate should satisfy

$$
\begin{aligned}
F\left(|\boldsymbol{q}|_{\min },\left|\boldsymbol{p}_{2}\right|_{\text {min }}\right)= & \sqrt{\left(\left|\boldsymbol{p}_{2}\right|_{\min }-E_{1}\right)^{2}+\tilde{m}_{4}^{* 2}} \\
& -\sqrt{\left|\boldsymbol{p}_{2}\right|_{\min }^{2}+m_{2}^{* 2}} \\
& +U_{4}-U_{2}-E_{1}<0
\end{aligned}
$$

if $\tilde{m}_{4}^{*}<m_{2}^{*}$, or

$$
F\left(|\boldsymbol{q}|_{\min }, \infty\right)=U_{4}-U_{2}-2 E_{1}<0,
$$

if $\tilde{m}_{4}^{*} \geq m_{2}^{*}$.

If the maximum of $F\left(|\boldsymbol{q}|_{\min },\left|\boldsymbol{p}_{2}\right|\right)$ is negative, i.e.,

$$
\max \left\{F\left(|\boldsymbol{q}|_{\min }, 0\right), F\left(|\boldsymbol{q}|_{\min }, \infty\right)\right\} \leq 0,
$$

there will be no constraint on $\left|\boldsymbol{p}_{2}\right|$. Otherwise, the bounds are given by the positive solutions of $F\left(|\boldsymbol{q}|_{\min },\left|\boldsymbol{p}_{2}\right|\right)=0$. We have checked that the lower bound of $\left|\boldsymbol{p}_{2}\right|$ is given by

$$
\left|\boldsymbol{p}_{2}\right|^{\text {low }}=\frac{E_{1} E_{s q} \pm \sqrt{E^{\prime 2} E_{q u}}}{2\left(E_{1}^{2}-E^{\prime 2}\right)}
$$

where "+" and "-" correspond to cases with $U_{4}-U_{2}-$ $E_{1} \geq 0$ and $U_{4}-U_{2}-E_{1}<0$, respectively, and

$$
\begin{aligned}
E^{\prime} & =E_{1}+U_{2}-U_{4}, \\
E_{s q} & =E_{1}^{2}+\tilde{m}_{4}^{* 2}-m_{2}^{* 2}-E^{\prime 2}, \\
E_{q u} & =E_{s q}^{2}+4 m_{2}^{* 2}\left(E_{1}^{2}-E^{\prime 2}\right) .
\end{aligned}
$$

In the special case where $E_{1}^{2}=E^{\prime 2}$, the possible solution is

$$
\left|\boldsymbol{p}_{2}\right|^{\text {low }}=\frac{E_{s q}^{2}-4 E^{\prime 2} m_{2}^{* 2}}{4 E_{1} E_{s q}} .
$$

(b) Upper/lower bounds of $|\boldsymbol{q}|$ :

For a given value of $\left|\boldsymbol{p}_{2}\right|$ between $\left|\boldsymbol{p}_{2}\right|^{\text {low }}$ and $\left|\boldsymbol{p}_{2}\right|^{\text {up }}$, the bounds of $|\boldsymbol{q}|$ can be determined by equations $q_{0}^{H, \text { min }}=$ $q_{0}^{L, \text { max }}$ or $q_{0}^{H, \text { max }}=q_{0}^{L, \text { min }}$, see Eq. (C1), and we obtain

$$
\begin{aligned}
|\boldsymbol{q}|^{\text {low }} & =\left|\frac{B_{q}+\sqrt{B_{q}^{2}-4 A_{q} C_{q}}}{2 A_{q}}\right|, \\
|\boldsymbol{q}|^{\text {up }} & =\frac{-B_{q}+\sqrt{B_{q}^{2}-4 A_{q} C_{q}}}{2 A_{q}},
\end{aligned}
$$

where

$$
\begin{aligned}
& A_{q}=4\left[E_{q}^{2}-\left(E_{1}-\left|\boldsymbol{p}_{2}\right|\right)^{2}\right], \\
& B_{q}=4\left[E_{1}\left(E_{q s q}-E_{q}^{2}\right)-\left|\boldsymbol{p}_{2}\right|\left(E_{q s q}+E_{q}^{2}\right)\right], \\
& C_{q}=4 E_{q}^{2}\left(\left|\boldsymbol{p}_{2}\right|^{2}+m_{4}^{* 2}\right)-\left(E_{q s q}-E_{q}^{2}\right)^{2},
\end{aligned}
$$

with $E_{q}=E_{1}+E_{2}-U_{4}$ and $E_{q s q}=E_{1}^{2}+m_{3}^{2}-m_{4}^{* 2}-$ $\left|\boldsymbol{p}_{2}\right|^{2}$.

(c) Upper/lower bounds of $\cos \theta_{q 2}$ :

Once fixing the values of $\boldsymbol{p}_{2}$ and $|\boldsymbol{q}|$, the minimum and maximum of $q_{0}$ are simply

$$
\begin{aligned}
& q_{0}^{\min }=\max \left\{q_{0}^{H, \min }, q_{0}^{L, \min }\right\}, \\
& q_{0}^{\max }=\min \left\{q_{0}^{H, \max }, q_{0}^{L, \max }\right\} .
\end{aligned}
$$

Since $q_{0}=E_{4}-E_{2}=\sqrt{\left|\boldsymbol{p}_{2}+\boldsymbol{q}\right|^{2}+m_{4}^{* 2}}+U_{4}-E_{2}$, the bounds of $\cos \theta_{q 2}$ can be obtained as

$$
\begin{aligned}
& \cos \theta_{q 2}^{\text {low }}=\max \left\{-1, \frac{\left(q_{0}^{\min }+E_{2}-U_{4}\right)^{2}-m_{4}^{* 2}-\left|\boldsymbol{p}_{2}\right|^{2}-|\boldsymbol{q}|^{2}}{2\left|\boldsymbol{p}_{2}\right||\boldsymbol{q}|}\right\}, \\
& \cos \theta_{q 2}^{\text {up }}=\min \left\{1, \frac{\left(q_{0}^{\max }+E_{2}-U_{4}\right)^{2}-m_{4}^{* 2}-\left|\boldsymbol{p}_{2}\right|^{2}-|\boldsymbol{q}|^{2}}{2\left|\boldsymbol{p}_{2}\right||\boldsymbol{q}|}\right\} .
\end{aligned}
$$

(d) Upper/lower bounds of $\phi_{q 2}$ :

$$
\phi_{q 2}^{\text {low }}=0, \quad \phi_{q 2}^{\text {up }}=2 \pi .
$$

In summary, Eqs. (C5) or (C6) determines the threshold of neutrino energy $E_{1}$, and Eqs. (C8) and (C10), (C11), (C14), and (C15) give the upper/lower bounds for the integration variables $\left|E_{2}\right|,|\boldsymbol{q}|, \cos \theta_{q 2}$ and $\phi_{q 2}$, respectively.

\section{Bounds for inverse decay}

The bounds of the integration variables for inverse decay, $\nu_{1}+N_{2}+l_{3} \rightarrow N_{4}$, or $\nu_{1}+l_{2}+\nu_{3} \rightarrow l_{4}$, can be obtained similarly as for neutrino absorption or scattering, as discussed above. For inverse decay, we have different definitions of $\boldsymbol{q}$ and $q_{0}$ with $\boldsymbol{q}=\boldsymbol{p}_{4}-\boldsymbol{p}_{2}=\boldsymbol{p}_{1}+\boldsymbol{p}_{3}$ and $q_{0}=E_{4}-E_{2}=E_{1}+E_{3}$. Correspondingly, the maximal and minimal values of $q_{0}$ shown in Eq. (C1) turn to

$$
\begin{aligned}
& q_{0, \mathrm{dec}}^{H, \max }=\sqrt{\left(\left|\boldsymbol{p}_{2}\right|+|\boldsymbol{q}|\right)^{2}+m_{4}^{* 2}}+U_{4}-E_{2}, \\
& q_{0, \mathrm{dec}}^{H, \min }=\sqrt{\left(\left|\boldsymbol{p}_{2}\right|-|\boldsymbol{q}|\right)^{2}+m_{4}^{* 2}}+U_{4}-E_{2},
\end{aligned}
$$




$$
\begin{aligned}
& q_{0, \text { dec }}^{L, \max }=E_{1}+\sqrt{\left.E_{1}+|\boldsymbol{q}|\right)^{2}+m_{3}^{2}}, \\
& q_{0, \mathrm{dec}}^{L, \min }=E_{1}+\sqrt{\left(E_{1}-|\boldsymbol{q}|\right)^{2}+m_{3}^{2}} .
\end{aligned}
$$

We note that $q_{0, \text { dec }}^{L, \text { max }}>q_{0, \text { dec }}^{H, \text { min }}$ is guaranteed at $|\boldsymbol{q}| \rightarrow \infty$, so we only need to consider the requirement $q_{0, \operatorname{dec}}^{H, \max } \geq$ $q_{0, \mathrm{dec}}^{L, \min }$. We choose the same integration variables as for neutrino scattering/absorption and take the same steps to determine their bounds.

(a) Allowed range of $E_{1}$ and upper/lower bounds of $E_{2}$ or $\left|\boldsymbol{p}_{2}\right|$ :

If $\tilde{m}_{4 \text {,dec }}^{*}=m_{4}^{*}-m_{3}>m_{2}^{*}$, the kinematically allowed $E_{1}$ should satisfy

$$
\begin{aligned}
F_{\mathrm{dec}}\left(|\boldsymbol{q}|_{\max },\left|\boldsymbol{p}_{2}\right|_{\max }\right)= & \sqrt{\left(\left|\boldsymbol{p}_{2}\right|_{\max }+E_{1}\right)^{2}+\tilde{m}_{4, \mathrm{dec}}^{* 2}} \\
& -\sqrt{\left|\boldsymbol{p}_{2}\right|_{\max }^{2}+m_{2}^{* 2}} \\
& +U_{4}-U_{2}-E_{1}>0,
\end{aligned}
$$

where $F_{\text {dec }}\left(|\boldsymbol{q}|,\left|\boldsymbol{p}_{2}\right|\right)=q_{0, \text { dec }}^{H, \max }-q_{0, \text { dec }}^{L, \text { in }}$, and

$$
\begin{gathered}
|\boldsymbol{q}|_{\max }=\frac{m_{3}\left|\boldsymbol{p}_{2}\right|+m_{4}^{*} E_{1}}{\tilde{m}_{4, \mathrm{dec}}^{*}}, \\
\left|\boldsymbol{p}_{2}\right|_{\max }=\frac{m_{2}^{*} E_{1}}{\tilde{m}_{4, \mathrm{dec}}^{*}-m_{2}^{*}} .
\end{gathered}
$$

While for $\tilde{m}_{4, \mathrm{dec}}^{*} \leq m_{2}^{*}$, one should have

$$
F_{\text {dec }}\left(|\boldsymbol{q}|_{\max }, \infty\right)=U_{4}-U_{2}>0 .
$$

If the minimum of $F_{\mathrm{dec}}\left(|\boldsymbol{q}|_{\max },\left|\boldsymbol{p}_{2}\right|\right)$ is positive, i.e.,

$$
\min \left\{F_{\mathrm{dec}}\left(|\boldsymbol{q}|_{\max }, 0\right), F_{\mathrm{dec}}\left(|\boldsymbol{q}|_{\max }, \infty\right)\right\} \geq 0,
$$

there will be no constraint on $\left|\boldsymbol{p}_{2}\right|$. Otherwise, the lower bound of $\left|\boldsymbol{p}_{2}\right|$ is given by the positive solution of $F_{\text {dec }}\left(|\boldsymbol{q}|,\left|\boldsymbol{p}_{2}\right|\right)=0$ :

$$
\left|\boldsymbol{p}_{2}\right|^{\text {low }}=\frac{E_{1} E_{\mathrm{sq}, \mathrm{dec}} \pm \sqrt{E_{\mathrm{dec}}^{\prime 2} E_{\mathrm{qu}, \mathrm{dec}}}}{2\left(E_{\mathrm{dec}}^{\prime 2}-E_{1}^{2}\right)},
$$

where + and - correspond to cases with $U_{4}-U_{2}-E_{1} \geq 0$ and $U_{4}-U_{2}-E_{1}<0$, respectively, and

$$
\begin{aligned}
E_{\mathrm{dec}}^{\prime} & =E_{1}+U_{2}-U_{4}, \\
E_{\mathrm{sq}, \mathrm{dec}} & =E_{1}^{2}+\tilde{m}_{4, \mathrm{dec}}^{* 2}-m_{2}^{* 2}-E_{\mathrm{dec}}^{\prime 2}, \\
E_{\mathrm{qu}, \mathrm{dec}} & =E_{\mathrm{sq}, \mathrm{dec}}^{2}+4 m_{2}^{* 2}\left(E_{1}^{2}-E_{\mathrm{dec}}^{\prime 2}\right) .
\end{aligned}
$$

In the case where $E_{1}^{2}=E_{\mathrm{dec}}^{2}$,

$$
\left|\boldsymbol{p}_{2}\right|^{\text {low }}=\frac{4 E^{\prime 2} m_{2}^{* 2}-E_{s q}^{2}}{4 E_{1} E_{s q}} .
$$

(b) Upper/lower bounds of $|\boldsymbol{q}|$ :

$$
\begin{aligned}
|\boldsymbol{q}|^{\text {low }} & =\left|\frac{B_{q, \mathrm{dec}}+\sqrt{B_{q, \mathrm{dec}}^{2}-4 A_{q, \mathrm{dec}} C_{q, \mathrm{dec}}}}{2 A_{q, \mathrm{dec}}}\right| \\
|\widetilde{\boldsymbol{q}}|^{\text {up }} & =\frac{-B_{q, \mathrm{dec}}+\sqrt{B_{q, \mathrm{dec}}^{2}-4 A_{q, \mathrm{dec}} C_{q, \mathrm{dec}}}}{2 A_{q, \mathrm{dec}}},
\end{aligned}
$$

where

$A_{q, \mathrm{dec}}=4\left[E_{q}^{2}-\left(E_{1}+\left|\boldsymbol{p}_{2}\right|\right)^{2}\right]$,

$B_{q, \mathrm{dec}}=4\left[E_{1}\left(E_{q s q}-E_{q}^{2}\right)+\left|\boldsymbol{p}_{2}\right|\left(E_{q s q}+E_{q}^{2}\right)\right]$,

$C_{q, \mathrm{dec}}=4 E_{q}^{2}\left(\left|\boldsymbol{p}_{2}\right|^{2}+m_{4}^{* 2}\right)-\left(E_{q s q}-E_{q}^{2}\right)^{2}$,

with $E_{q}$ and $E_{q s q}$ given below Eq. (C12). It should emphasized that the upper bound $|\boldsymbol{q}|^{\text {up }}=\mid \widetilde{\boldsymbol{q}}^{\text {up }}$ only if $|\widetilde{q}|^{\text {up }}>0$. Otherwise, there will be no upper bound for $|\boldsymbol{q}|$.

(c) Upper/lower bounds of $\cos \theta_{q 2}$ :

$\cos \theta_{q 2}^{\text {low }}=\max \left\{-1, \frac{\left(q_{0, \text { dec }}^{\min }+E_{2}-U_{4}\right)^{2}-m_{4}^{* 2}-\left|\boldsymbol{p}_{2}\right|^{2}-|\boldsymbol{q}|^{2}}{2\left|\boldsymbol{p}_{2}\right||\boldsymbol{q}|}\right\}$,

$\cos \theta_{q 2}^{\text {up }}=\min \left\{1, \frac{\left(q_{0, \mathrm{dec}}^{\max }+E_{2}-U_{4}\right)^{2}-m_{4}^{* 2}-\left|\boldsymbol{p}_{2}\right|^{2}-|\boldsymbol{q}|^{2}}{2\left|\boldsymbol{p}_{2}\right||\boldsymbol{q}|}\right\}$,

where $\quad q_{0, \text { dec }}^{\min }=\max \left\{q_{0, \operatorname{dec}}^{H, \min }, q_{0, \operatorname{dec}}^{L, \min }\right\} \quad$ and $\quad q_{0, \text { dec }}^{\max }=$ $\min \left\{q_{0, \operatorname{dec}}^{H, \max }, q_{0, \mathrm{dec}}^{L, \max }\right\}$, with $q_{0, \mathrm{dec}}^{H / L, \max / \min }$ shown in Eq. (C16).

(d) Upper/lower bounds of $\phi_{q 2}$ :

$$
\phi_{q 2}^{\mathrm{low}}=0, \quad \phi_{q 2}^{\mathrm{up}}=2 \pi .
$$


[1] H.-T. Janka, Annu. Rev. Nucl. Part. Sci. 62, 407 (2012).

[2] A. Burrows, S. Reddy, and T. A. Thompson, Nucl. Phys. A777, 356 (2006).

[3] H.-T. Janka, K. Langanke, A. Marek, G. Martínez-Pinedo, and B. Müller, Phys. Rep. 442, 38 (2007).

[4] A. Burrows, Rev. Mod. Phys. 85, 245 (2013).

[5] G. Martínez-Pinedo, T. Fischer, K. Langanke, A. Lohs, A. Sieverding, and M.-R. Wu, Neutrinos and their impact on core-collapse supernova nucleosynthesis, in Handbook of Supernovae, edited by A. W. Alsabti and P. Murdin (Springer International Publishing, Cham, 2016), pp. 18051841.

[6] T. Melson, H.-T. Janka, R. Bollig, F. Hanke, A. Marek, and B. Müller, Astrophys. J. 808, L42 (2015).

[7] P. J. Schinder and S. L. Shapiro, Astrophys. J. Suppl. Ser. 50, 23 (1982).

[8] S. W. Bruenn, Astrophys. J. Suppl. Ser. 58, 771 (1985).

[9] A. Mezzacappa and S. W. Bruenn, Astrophys. J. 410, 740 (1993).

[10] S. Hannestad and G. Raffelt, Astrophys. J. 507, 339 (1998).

[11] S. Reddy, M. Prakash, and J. M. Lattimer, Phys. Rev. D 58, 013009 (1998).

[12] S. Reddy, M. Prakash, J. M. Lattimer, and J. A. Pons, Phys. Rev. C 59, 2888 (1999).

[13] C. J. Horowitz, Phys. Rev. D 65, 043001 (2002).

[14] R. Buras, H.-T. Janka, M. T. Keil, G. G. Raffelt, and M. Rampp, Astrophys. J. 587, 320 (2003).

[15] C. J. Horowitz and A. Schwenk, Phys. Lett. B 642, 326 (2006).

[16] A. Juodagalvis, K. Langanke, W. R. Hix, G. MartínezPinedo, and J. Sampaio, Nucl. Phys. A848, 454 (2010).

[17] L. F. Roberts, S. Reddy, and G. Shen, Phys. Rev. C 86, 065803 (2012).

[18] A. Bartl, C. J. Pethick, and A. Schwenk, Phys. Rev. Lett. 113, 081101 (2014).

[19] C. J. Horowitz, O. L. Caballero, Z. Lin, E. O'Connor, and A. Schwenk, Phys. Rev. C 95, 025801 (2017).

[20] L. F. Roberts and S. Reddy, Phys. Rev. C 95, 045807 (2017).

[21] P. F. Bedaque, S. Reddy, S. Sen, and N. C. Warrington, Phys. Rev. C 98, 015802 (2018).

[22] G. Guo and G. Martínez-Pinedo, Astrophys. J. 887, 58 (2019).

[23] M. Rampp and H.-T. Janka, Astron. Astrophys. 396, 361 (2002).

[24] M. Liebendoerfer, M. Rampp, H.-T. Janka, and A. Mezzacappa, Astrophys. J. 620, 840 (2005).

[25] R. Buras, M. Rampp, H.-T. Janka, and K. Kifonidis, Astron. Astrophys. 447, 1049 (2006).

[26] T. Fischer, S. C. Whitehouse, A. Mezzacappa, F.-K. Thielemann, and M. Liebendrfer, Astron. Astrophys. 517, A80 (2010).

[27] G. Martínez-Pinedo, T. Fischer, A. Lohs, and L. Huther, Phys. Rev. Lett. 109, 251104 (2012).

[28] E. O'Connor, Astrophys. J. Suppl. Ser. 219, 24 (2015).

[29] A. Bartl, R. Bollig, H.-T. Janka, and A. Schwenk, Phys. Rev. D 94, 083009 (2016).
[30] T. Fischer, Astron. Astrophys. 593, A103 (2016).

[31] L. F. Roberts, C. D. Ott, R. Haas, E. P. O'Connor, P. Diener, and E. Schnetter, Astrophys. J. 831, 98 (2016).

[32] K. Kotake, T. Takiwaki, T. Fischer, K. Nakamura, and G. Martínez-Pinedo, Astrophys. J. 853, 170 (2018).

[33] T. Fischer, G. Guo, A. A. Dzhioev, G. Martínez-Pinedo, M.-R. Wu, A. Lohs, and Y.-Z. Qian, Phys. Rev. C 101, 025804 (2020).

[34] R. Bollig, H.-T. Janka, A. Lohs, G. Martínez-Pinedo, C. J. Horowitz, and T. Melson, Phys. Rev. Lett. 119, 242702 (2017).

[35] A. Burrows and R.F. Sawyer, Phys. Rev. C 58, 554 (1998).

[36] A. Burrows and R. F. Sawyer, Phys. Rev. C 59, 510 (1999).

[37] C. J. Horowitz and M. A. Perez-Garcia, Phys. Rev. C 68, 025803 (2003).

[38] B. Fore and S. Reddy, Phys. Rev. C 101, 035809 (2020).

[39] W. R. Yueh and J. R. Buchler, Astrophys. Space Sci. 39, 429 (1976).

[40] W. R. Yueh and J. R. Buchler, Astrophys. Space Sci. 41, 221 (1976).

[41] A. Lohs, Ph.D. thesis, Technische Universität Darmstadt, 2015.

[42] G. Fuller, W. Fowler, and M. J. Newman, Astrophys. J. 293, 1 (1985).

[43] G. Martínez-Pinedo, Y. H. Lam, K. Langanke, R. G. T. Zegers, and C. Sullivan, Phys. Rev. C 89, 045806 (2014).

[44] H. Shen, H. Toki, K. Oyamatsu, and K. Sumiyoshi, Nucl. Phys. A637, 435 (1998).

[45] S. Typel and H. H. Wolter, Nucl. Phys. A656, 331 (1999).

[46] S. Typel, Phys. Rev. C 71, 064301 (2005).

[47] M. Hempel and J. Schaffner-Bielich, Nucl. Phys. A837, 210 (2010).

[48] G. Shen, C. J. Horowitz, and S. Teige, Phys. Rev. C 82, 015806 (2010).

[49] S. Typel, G. Ropke, T. Klahn, D. Blaschke, and H. H. Wolter, Phys. Rev. C 81, 015803 (2010).

[50] S. Furusawa, S. Yamada, K. Sumiyoshi, and H. Suzuki, Astrophys. J. 738, 178 (2011).

[51] G. Shen, C. J. Horowitz, and S. Teige, Phys. Rev. C 83, 035802 (2011).

[52] G. Shen, C. J. Horowitz, and E. O'Connor, Phys. Rev. C 83, 065808 (2011).

[53] M. Hempel, T. Fischer, J. Schaffner-Bielich, and M. Liebendorfer, Astrophys. J. 748, 70 (2012).

[54] A. W. Steiner, M. Hempel, and T. Fischer, Astrophys. J. 774, 17 (2013).

[55] J. M. Lattimer and F. D. Swesty, Nucl. Phys. A535, 331 (1991).

[56] L. B. Leinson and A. Pérez, Phys. Lett. B 518, 15 (2001).

[57] L. B. Leinson, Nucl. Phys. A707, 543 (2002).

[58] T. Hahn, Comput. Phys. Commun. 168, 78 (2005).

[59] https://wwwmpa.mpa-garching.mpg.de/ccsnarchive.

[60] S. Woosley and A. Heger, Phys. Rep. 442, 269 (2007).

[61] M. T. Keil, G. G. Raffelt, and H.-T. Janka, Astrophys. J. 590, 971 (2003). 